\title{
DESARROLLO DE INSUMOS PARA LA TOMA DE DECISIONES DE CONSERVACIÓN EN LA CUENCA AMAZÓNICA OCCIDENTAL
}

\section{DECISION-MAKING INPUTS FOR THE CONSERVATION OF THE WESTERN AMAZON BASIN}

\author{
C. Josse ${ }^{1}$, B. Young ${ }^{2}$, R. Lyons-Smyth ${ }^{3}$, T. Brooks ${ }^{4}$, A. Frances ${ }^{5}$, P. Comer ${ }^{6}$, P. Petry ${ }^{7}$, H. Balslev ${ }^{8}$, B. \\ Bassuner $^{9}$, B. Goettsch ${ }^{10}$, J. Hak ${ }^{11}$, P. Jørgensen ${ }^{12}$, D. Larrea-Alcázar ${ }^{13}$, G. Navarro ${ }^{14}$, S. Saatchi ${ }^{15}$, A. Sanchez de \\ Lozada $^{16}$, J. C. Svenning ${ }^{17}$, L. A. Tovar ${ }^{18}$ y A. Moscoso ${ }^{19}$
}

\begin{abstract}
Resumen
El actual modelo de crecimiento económico que estimula a los países ricos en recursos a convertirse en proveedores de materias primas para un mercado global en expansión, está afectando gravemente la condición de grandes áreas naturales, tales como la cuenca occidental del Amazonas, una de las regiones con mayor biodiversidad del planeta. El proceso se acelerará a medida que esta región se vuelva más accesible con el desarrollo de la infraestructura de transporte, así como con la extracción de recursos no renovables. Disminuir estas presiones con el fin de salvaguardar la biodiversidad y los servicios ecosistémicos que presta a la humanidad requiere que los procesos de toma de decisiones se basen en datos relevantes y actualizados. En este sentido, se han aprovechado nuevos datos disponibles para varios grupos de especies, sistemas ecológicos acuáticos y terrestres, así como la distribución de las amenazas y los servicios de los ecosistemas, para medir y mapear a través de un área $>2$ millones de $\mathrm{km}^{2}$, el estado y las presiones de la biodiversidad y la respuesta de la conservación y los beneficios, usando las subcuencas como unidades de análisis. Esta información, organizada según el marco Presión-EstadoRespuesta-Beneficios, debe apoyar las decisiones en materia de inversiones en conservación y desarrollo sostenible y el seguimiento de sus impactos en el largo plazo.

Palabras clave: Amazonía Andina, amenazas, ecosistemas desprotegidos, ecosistemas amazónicos, especies amenazadas, planificación de conservación, rareza geográfica, servicios ambientales.
\end{abstract}

\begin{abstract}
Current economic growth models encourage natural resource-rich countries to become suppliers of raw materials for growing capital markets which severely affect conditions of large natural areas such as the western Amazon Basin, one of the most biodiverse regions on Earth. The process will accelerate as this region becomes more accessible with the development of transportation infrastructure as well as the extraction of non-renewable resources. Ameliorating these pressures in order to safeguard biodiversity and the ecosystem services it provides to humanity will require that decision-making processes are informed by relevant and up-to-date datasets. Here, we capitalize on newly available data for several species groups, aquatic and terrestrial ecological systems, and the distribution of threats and ecosystem services, to measure and map biodiversity state and pressure, and conservation response and benefits for sub-basins across a $>2$ million- $\mathrm{km}^{2}$ area. This information, organized according to the Pressure-StateResponse-Benefits framework, should support for decisions on conservation and sustainable development investments, and monitoring their impact over the long term.

Key words: Andes Amazon, Amazon ecosystems, conservation planning, ecosystem services, range size rarity, threatened species, threats, unprotected ecosystems.
\end{abstract}

\section{Introducción.}

La porción occidental de la cuenca amazónica se destaca por incluir la vertiente oriental de los Andes tropicales, abarcando así una región reconocida a nivel mundial y continental por su alta riqueza y nivel de endemismo de plantas vasculares, aves, anfibios y reptiles (Fjeldså \& Rahbek, 1998; Myers et al., 2000;
Olson \& Dinerstein, 2002; Rodrigues et al., 2004; Rodríguez-Mahecha et al., 2004; Brooks et al.,2006). Esta región también es clave a nivel global por la provisión de servicios ecosistémicos, concretamente el almacenamiento de carbono y la provisión de agua dulce (Saatchi et al., 2011; Baccini et al., 2012; Larsen et al., 2011). Sin embargo, la compleja 
distribución espacial de las características geomorfológicas, el clima, los recursos biológicos y las poblaciones humanas y sus actividades en esta región tan heterogénea y extensa, inevitablemente conducen a una distribución irregular de las presiones y las necesidades de conservación. De ahí la necesidad de identificar la importancia relativa de ciertas áreas con respecto a determinados criterios con el fin de promover decisiones orientadas a la sustentabilidad.

La creciente demanda por productos primarios y el alto precio de los combustibles generan modelos productivos que son la fuente de impactos a la integridad ecológica de los sistemas amazónicos, siendo el principal la deforestación a causa de la agricultura y ganadería (Nepstad et al., 2008; Raisg 2012), seguido de efectos de contaminación de agua, tierra y aire por la extracción petrolera y minera (Finer \& Orta Martínez, 2010; Swenson et al., 2011; RAISG, 2012). El modelo económico demanda a su vez infraestructura de transporte a nuevas áreas para desarrollar estas actividades, lo que conlleva facilitar el acceso a la explotación forestal, generando más fragmentación y un empobrecimiento en la estructura y composición de los bosques en pie (Killeen, 2007; Nepstad et al., 2008; RAISG, 2012). Al combinar este escenario actual con las predicciones de los modelos de cambio climático, las interacciones detectadas tienen un impacto potencial tan grande que el futuro de la Amazonía es un tema de seria consideración (Barlow \& Peres, 2008; Betts et al., 2008; Li et al., 2008; PNUMA \& OTCA, 2009). Estos antecedentes han renovado el interés de diferentes actores en la región, entre ellos corporaciones privadas, bancos multilaterales, cooperación bilateral, fundaciones filantrópicas y los gobiernos nacionales, por identificar para la región en su conjunto las áreas claves para la conservación de los distintos componentes de la biodiversidad y sus beneficios. De ahí surge la pregunta, ¿dónde dentro de esta vasta región deben invertirse los recursos disponibles para la conservación y dónde deben aplicarse salvaguardas que permitan un desarrollo sustentable? Para contestar este tipo pregunta, se han desarrollado diferentes metodologías de priorización y planificación (véase Groves, 2003 para una revisión exhaustiva). El resalta como hitos en este tema la incepción del concepto de análisis de representatividad (Edwards et al., 1993), la planificación al interior de las ecoregiones (Groves et al., 2000) y la planificación sistemática de la conservación (Margules \& Pressey, 2000). Adicionalmente, con el creciente interés en el tema de la mantención de los servicios ecosistémicos de importancia regional y global (Millenium Ecosystem Assessment, 2005) y esquemas de pago por estos servicios, la identificación de áreas clave en la provisión de los mismos cobra especial importancia, ya sea por la posibilidad de incluirlas en análisis de costo beneficio o por la potencial convergencia de sitios de alto valor de biodiversidad y alto valor de servicios (Turner et al., 2012).

Todos estos métodos tienen componentes del esquema Presión-Estado-Respuesta-Beneficio, que como marco lógico facilita identificar los vínculos causales entre los componentes y permite desarrollar indicadores para el monitoreo tomando en cuenta los vínculos de causalidad (Figura 1) (UNEP-WCMC, 2009; Sparks et al., 2011). La adopción de este marco fue discutida en el seno de la Convención para la Diversidad Biológica (CBD, 2010) para hacer el seguimiento a las metas fijadas en Aichi (COP Aichi, 2010). En este estudio se adopta este marco, donde la presión equivale a los impactos y amenazas a la biodiversidad a causa de las acciones humanas, el estado equivale a la biodiversidad misma (representada por los vacíos en la conservación de especies y ecosistemas amenazados), la respuesta equivale a las políticas y acciones de conservación que implican costos, y el beneficio a los servicios que la naturaleza devuelve a la sociedad, en este caso en la forma de aprovisionamiento de agua y potencial REDD ("Reducing Emissions from Deforestation and Forest Degradation”) de carbono.

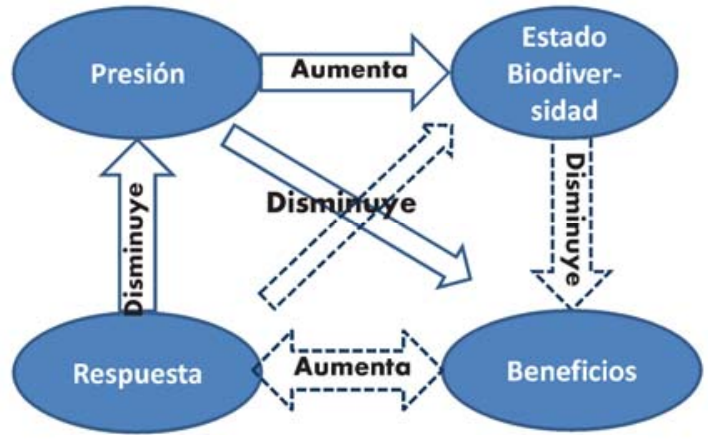

Figura 1. Diagrama de las relaciones entre indicadores de áreas temáticas claves para el monitoreo de metas e impactos de los esfuerzos de conservación.

Para este estudio se han aprovechado conjuntos de datos recientemente disponibles y comprehensivos para múltiples grupos de especies, sistemas ecológicos terrestres y acuáticos, la distribución de amenazas y los beneficios provenientes de ecosistemas en términos de flujos hidrológicos y almacenamiento de carbono. Aquí se describen los análisis realizados para calificar la importancia relativa por sub-cuenca de cada uno de estos componentes. A la vez, se ponen los datos a disposición pública para apoyar los esfuerzos de planificación de entidades que trabajan en la región.

\section{Materiales y métodos.}

1 Contexto geográfico y unidades de análisis 
El área de estudio comprende la cuenca amazónica en Colombia, Ecuador, Perú y Bolivia. Delimitada en base a los criterios político e hidrológico, cubre 2190 $000 \mathrm{~km}^{2}$ desde los $6250 \mathrm{msnm}$ hasta las fronteras nacionales en la llanura amazónica (Figura 2).

La cobertura de las áreas protegidas en esta región alcanza un $17 \%$ del área de estudio según la información de las fuentes nacionales actualizadas al 2011 y proporcionadas por colaboradores (Fundación Natura, Colombia; EcoCiencia, Ecuador; Geomáticos Consultores, Perú y FAN, Bolivia) y considerando las áreas nacionales en las categorías I-V según UICN, así como áreas naturales de protección regional (Figura 2). Se utilizó esta información para desarrollar análisis de vacíos cuyo uso se detalla más adelante.

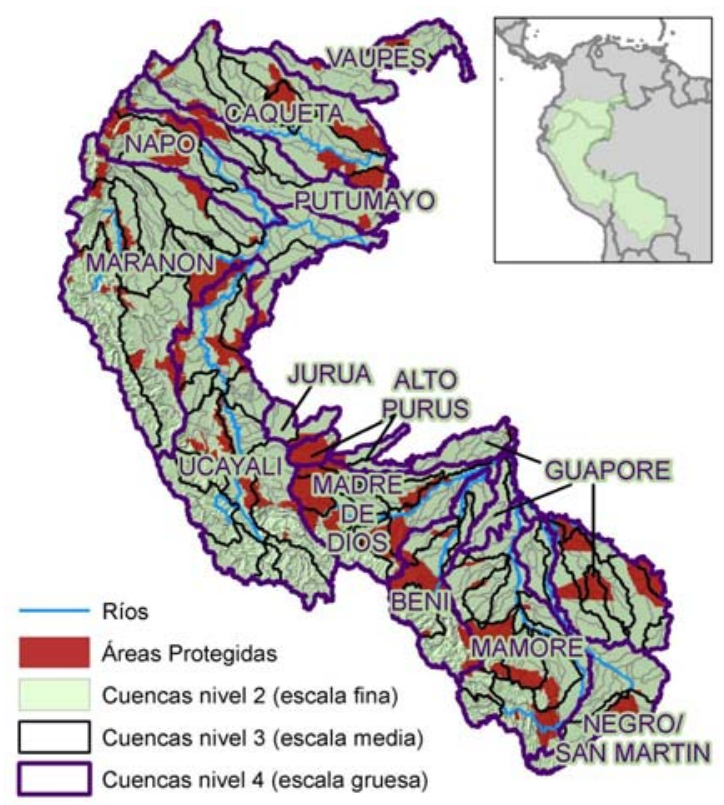

Figura 2. Mapa del área del estudio mostrando la organización de unidades de cuenca adoptada para los análisis y las áreas protegidas.

Las unidades de análisis utilizadas en el estudio son las cuencas hidrográficas delimitadas por The Nature Conservancy (TNC) (Petry \& Sotomayor, 2009) según el método de Fitzhugh (2005), que genera unidades de cuenca a diferentes tamaños establecidos por el usuario, pero siempre manteniendo la estructura espacialmente anidada de los diferentes niveles a los que corresponden las clases de tamaño (Figura 2). Los tamaños de cuenca derivados por TNC para esta clasificación y aplicados al área del proyecto se detallan en la Tabla 1. La razón de adoptar esta unidad es que a diferencia de jurisdicciones políticoadministrativas, por ejemplo, representan unidades naturales discretas. Además, por su estructura anidada o jerárquica permiten analizar y presentar resultados a diferentes niveles de cuenca. Cada unidad de cuenca tiene un código único en la base de datos cuya composición denota su pertenencia a una cuenca específica del nivel superior. Los análisis que se presentan fueron realizados usando las cuencas de escala media (nivel 3), representadas por 66 unidades en el área de estudio. Para controlar el efecto de la variación del tamaño entre cuencas del mismo nivel, todos los análisis y cálculos se realizaron usando hexágonos de tamaño idéntico (4 km de diámetro) y los resultados se promediaron por unidad de cuenca.

Tabla 1. Niveles, tamaño y número de unidades de sub-cuencas y cuencas en la vertiente amazónica de Colombia, Ecuador, Perú y Bolivia.

\begin{tabular}{lcr}
\hline Nivel (escala) & Tamaño $\left(\mathrm{km}^{2}\right)$ & $\begin{array}{c}\text { Número de } \\
\text { unidades }\end{array}$ \\
\hline 1 (muy fina) & $100-1000$ & 3990 \\
2 (fina) & $1000-10000$ & 448 \\
3 (media) & $10000-100000$ & 66 \\
4 (gruesa) & $100000-1000000$ & 13 \\
\hline
\end{tabular}

2 Biodiversidad.

2.1 Rareza geográfica de especies amenazadas.

Animales y Plantas .- Para los animales y plantas examinados, se cuantificó el valor de la biodiversidad como la rareza geográfica total promedio por cuenca de especies amenazadas y no protegidas según lo planteado por Brooks et al. (2006). Se incluyen en el análisis, entonces, todos los grupos que han sido evaluados en una forma comprehensiva según los criterios de la Lista Roja de la UICN (2001) y para los cuales existen mapas de distribución. Los mamíferos, aves y anfibios cuentan con esta información al nivel global (Stuart et al., 2004; Schipper et al., 2008; Vié et al., 2008), pero ningún otro grupo animal reúne estos requisitos. Para plantas, el único grupo con un análisis global y un número significativo de especies distribuidas en el área del estudio es Cactaceae (Goettsch et al., en preparación). Sin embargo, tres otras familias (Arecaceae, Brunelliaceae y Passifloraceae), han sido evaluadas al nivel nacional según los criterios de la UICN (Gärdenfors et al., 2001), en los cuatro países donde se ubica el área del estudio y por lo tanto se incluyó también estos grupos en el análisis (Calderón et al., 2005; León et al., 2007; León-Yánez et al., 2011; Ministerio de Medio Ambiente y Agua, 2012). Se reconoce que los grupos de animales analizados cuentan con más especies que los grupos de plantas. También se podría debatir si este conjunto de grupos de animales y plantas es el más representativo de la región andina. No obstante, los grupos que se incluyeron son, a nuestro conocimiento, los únicos con información sobre riesgo de extinción y distribución disponibles en la actualidad. Por lo tanto, se presentan los resultados separados por grupo taxonómico además de un resultado integral de todos los grupos.

Para los tres grupos de vertebrados y cuatro de plantas, el primer paso fue recopilar una lista de especies amenazadas, definida como las especies 
asignadas a las categorías de Vulnerable, En Peligro y En Peligro Crítico. Se consideró solamente especies y no rangos infraespecíficos. Luego se sobrepuso el mapa de áreas protegidas con el mapa de distribución de cada especie y se calculó el porcentaje de la distribución coincidente con la extensión de las áreas protegidas. Se utilizó la relación propuesta por Rodrigues et al. (2004) para determinar las especies no protegidas. Las especies con distribuciones de más de 250000 $\mathrm{km}^{2}$ y que tienen menos de $10 \%$ de su distribución representada al interior de las áreas protegidas fueron consideradas como no protegidas, junto con aquellas especies con distribuciones menores de $1000 \mathrm{~km}^{2}$ y menos de $100 \%$ de su distribución representada dentro de áreas protegidas. El umbral varía entre $100 \%$ y $10 \%$ en forma lineal para las especies con distribuciones entre $1000 \mathrm{~km}^{2}$ y $250000 \mathrm{~km}^{2}$ (Rodrigues et al., 2004).

Para los vertebrados, se usó los mapas de distribución publicados (BirdLife International \& NatureServe, 2012; UICN, 2012). Se usaron los mapas en Bjorholm et al. (2005) para las Arecaceae, Goettsch et al. (datos no publicados) para las Cactaceae y Jørgensen \& Bassuner (datos no publicados) para las Passifloraceae. No existían mapas en forma de polígono para las Brunelliaceae, entonces se crearon mapas para esas especies utilizando los límites de los sistemas ecológicos (Josse et al., 2009) donde las especies fueron colectadas. Se unieron las distribuciones de todos los sistemas ecológicos (con excepción de los sistemas antropogénicos) y luego se cortó el resultado según la distribución altitudinal conocida para la especie y los límites norte y sur de las localidades donde la especie ha sido registrada.

Se calculó la rareza geográfica de cada especie no protegida como el inverso de su distribución medida en $\mathrm{km}^{2}$ (también conocida como "rareza del tamaño de la distribución” Knapp, 2002). Este método da mayor peso a las especies con distribuciones pequeñas y refleja las reducidas opciones de conservación que presentan esas especies. El promedio de rareza geográfica para cada cuenca fue calculado usando los hexágonos. En cada hexágono, se sumó la rareza geográfica de todas las especies cuyas distribuciones se traslapan con el hexágono. Luego se calculó un promedio de esta rareza geográfica total en todos los hexágonos de cada cuenca. El promedio permitió comparaciones entre cuencas de tamaños diferentes pero del mismo nivel.

Muchas de las especies amenazadas de los grupos focales no son endémicas al área del estudio. En estos casos, se calculó el porcentaje de la distribución en áreas protegidas solamente tomando en cuenta la distribución dentro del área del estudio. Debido a que no se pudo conseguir una capa actualizada de los límites de las áreas protegidas de los países fuera del área de estudio donde se extienden las distribuciones de las especies amenazadas, no fue posible tomar en cuenta el nivel de protección fuera del área del estudio. En cambio, para todas las especies se tuvo sus distribuciones completas y entonces se calculó la rareza geográfica tomando en cuenta la distribución completa.

2.2 Ecosistemas terrestres: rareza geográfica e integridad.

Para obtener la cobertura de ecosistemas para toda el área de estudio, se unieron dos mapas desarrollados en base a la clasificación de sistemas ecológicos de NatureServe que, por lo tanto, comparten sus leyendas, el Mapa de ecosistemas de los Andes del norte y centro (Josse et al., 2009) y el Mapa de ecosistemas de la región Amazónica de los países de la CAN (Josse, 2009). La escala de producción de estos mapas fue 1:250 000 con una unidad mínima de mapeo de aproximadamente 25 hectáreas. En ambos 
casos reflejan la cobertura de los ecosistemas naturales al año 2009.

Para valorar la integridad de los ecosistemas terrestres se utilizó un modelo de condición del paisaje actual. Para generarlo se aplicó la herramienta Modelo de Condición del Paisaje (Comer \& Hak, 2012) que estima el impacto acumulado de los diferentes usos del suelo y presencia de infraestructura sobre la condición del paisaje. El resultado es una cobertura continua del área de estudio a $250 \mathrm{~m}$ de resolución, donde cada pixel tiene un valor entre 0 (mala condición) y 1 (excelente condición) que corresponde al índice de impacto. El modelo permite la atribución de pesos individuales a los elementos que constituyen las fuentes de impacto. Los pesos se asignan tanto para el sitio de su ubicación [intensidad], como en función de la distancia del impacto [alcance] (Tabla 2).

El primer paso fue identificar los ecosistemas con menos del $17 \%$ de su área de extensión dentro del sistema nacional de áreas protegidas. El umbral de 17\% proviene de las metas al 2020 del plan estratégico 2011-2020 del Convenio sobre la Diversidad Biológica (COP 10, 2010). Los ecosistemas así filtrados son los únicos que se utilizan para los análisis siguientes de rareza geográfica y rareza ponderada con integridad. De los 221 ecosistemas terrestres presentes en el área de estudio, 148 (67\%) califican como no protegidos.

Con estos ecosistemas, se hizo un análisis de rareza geográfica utilizando los hexágonos de $4 \mathrm{~km}$. Se obtuvo así un valor por hexágono que consiste en la suma de los valores correspondientes de la porción de cada ecosistema comprendida dentro del hexágono, dividida para su área total de distribución. Asi, hexágonos que contienen proporciones relativamente altas de ecosistemas no protegidos y muy restringidos en su distribución total, tuvieron valores mayores. La rareza geográfica por unidad de cuenca se calculó como el promedio de los valores de los hexágonos comprendidos dentro de cada unidad de cuenca.

En vista de que la integridad ecológica de los ecosistemas no es uniforme a través de toda su distribución en el área de estudio, se hizo un análisis de rareza geográfica ponderada con integridad, para el que se repitió el cálculo anterior, pero esta vez dividiendo el área en buena condición dentro de un hexágono por el área total de distribución del ecosistema. Se definió lo que equivale a buena condición de un ecosistema como áreas que coinciden con una condición de paisaje mayor a 0.6, umbral que se estableció en base al promedio de condición del paisaje para las áreas protegidas.

El resultado final de rareza geográfica de los ecosistemas terrestres fue un promedio de los dos resultados anteriores obtenidos por unidad de cuenca. Antes de calcular el promedio, cada valor se normalizó dividiéndolo para el valor máximo para esa métrica.

2.3 Ecosistemas acuáticos: rareza geográfica, integridad y conectividad hidrológica.

Los datos sobre ecosistemas acuáticos provienen de la clasificación derivada de la estratificación de variables físicas (fisiografía, geología, clima y características hidrográficas) (Téllez, 2011) y biogeográficas (Abell et al., 2008), que inciden en las características y composición biótica de los cursos de agua. A esta estratificación se aplicó un análisis de agrupación jerárquico para obtener la clasificación final a partir de los valores de disimilitud. Las unidades para representar la distribución geográfica de las 585 clases de ecosistemas acuáticos producto de la clasificación, son las unidades de cuenca del nivel 1 según el sistema de delineación de cuencas aplicado por Petry \& Sotomayor (2009) (Tabla 1). A estas unidades se aplicó un análisis de vacíos con el mismo umbral de $17 \%$ y se determinó que 374 (64\%) clases de ecosistemas acuáticos no están adecuadamente representadas en los sistemas de protección de áreas naturales. Estos fueron los ecosistemas utilizados en el análisis de rareza geográfica.

Para evaluar la conectividad hidrológica al nivel de cuenca se utilizaron los datos publicados por Finer \& Jenkins (2012) sobre la proliferación de represas hidroeléctricas planificadas para la región de Andes Amazonía. Estos datos incluyen las represas existentes a la fecha, de las cuales se usaron todas aquellas con una capacidad generadora superior a $2 \mathrm{MW}$.

Para estimar la rareza geográfica de los ecosistemas acuáticos no protegidos se utilizaron directamente las unidades de cuenca del nivel 1, donde la rareza del ecosistema es igual al área de la unidad de cuenca dividida para el área total de todas las cuencas del nivel 1 que corresponden a esa clase de ecosistema. Para obtener una medida de rareza geográfica de los ecosistemas acuáticos ponderada según su condición, la condición se calculó en base a dos criterios: la integridad y la conectividad hidrológica. La integridad de los ecosistemas acuáticos se evaluó con el mismo modelo de condición de paisaje aplicado a los ecosistemas terrestres.

Para estimar la conectividad se calificó a cada represa hidroeléctrica según su impacto potencial, donde a mayor capacidad generadora y ubicación en un curso de mayor orden, la calificación de impacto es más alta. La calificación de impacto inicial se hizo para cada cuenca de nivel 1 y luego la calificación de impacto para cada cuenca del nivel siguiente, se determinó mediante la suma de las puntuaciones de impacto de todas las presas situadas en las subcuencas de nivel 1. El valor de impacto de aquellas cuencas con cursos conectados aguas arriba o abajo fue sumado con el curso directamente afectado por la localización de la represa. Este esquema de valoración 
ponderó la proximidad, de modo que las represas cercanas afectarían más que represas ubicadas a mayores distancias. La calificación de la conectividad se calculó como el inverso de los valores de impacto obtenidos y luego se calificó la conectividad de las cuencas mayores (nivel 2, 3) con el promedio de las calificaciones de conectividad de las subcuencas correspondientes.

Adicionalmente, para cada cuenca de nivel 1 se obtuvo el valor medio de la condición de paisaje, que se promedió con la calificación de conectividad y luego se normalizó mediante la división por la calificación máxima obtenida. Este valor se multiplicó luego por la calificación de rareza geográfica de la cuenca, normalizada también de 0-1, para obtener así la calificación final de rareza ponderada por condición para los ecosistemas acuáticos. La calificación reportada para cuencas del nivel 3 representa un promedio ponderado por área de las calificaciones de las cuencas menores correspondientes.

3 Amenazas.

El análisis de amenazas se basa en información sobre distintos tipos de presiones antropogénicas, incluyendo tanto las infraestructuras (carreteras, poblados, pozos petroleros, minería y represas hidroeléctricas), como los usos e impactos derivados (agricultura, ganadería y deforestación) (Tabla 2).

La estimación del nivel de amenaza integró información sobre tres componentes: los cambios proyectados en la condición del paisaje, la conectividad hidrológica y la deforestación, a partir de la comparación entre el estado actual de cada uno de estos componentes y un escenario futuro (aproximadamente a 20 años).

Deterioro en la condición del paisaje. - Se utilizó la herramienta Modelo de Condición del Paisaje (Comer \& Hak, 2012), explicada arriba, para generar un modelo actual (2011) y uno futuro de la condición del paisaje, cubriendo toda el área de estudio a una resolución de 250 x 250 m (6.25 ha).

Para el modelo actual se incorporaron los insumos listados en la Tabla 2. El modelo futuro contempla los mismos impactos e insumos pero se añaden las carreteras proyectadas y áreas mineras disponibles para concesión, estimando un horizonte de 10-20 años. Se calculó el cambio en la condición restando el valor futuro del valor actual y, a partir de estos valores por pixel, se obtuvo el promedio para cada unidad de cuenca.

Cambio en la conectividad hidrológica. - Para este componente se aplicó el mismo criterio, según el que la amenaza está indicada por el deterioro entre la condición actual y un escenario futuro. Para el escenario actual se usó el mismo índice de impacto de las represas explicado arriba como un componente de la condición de los ecosistemas acuáticos. Para estimar el índice de impacto en un escenario futuro, se incluyeron en el análisis las represas planificadas más probables (Finer \& Jenkins, 2012), que suman 59 para toda el área, de modo que el valor utilizado es la diferencia entre el impacto futuro y el actual, calculado por cada cuenca del nivel 1 y promediado para la unidad de cuenca de nivel 3 , utilizando una ponderación por área.
Tabla 3. Variables utilizadas en el modelo de potencial de forestación en la cuenca amazónica. Las variables explicativas más de deforestación.

\begin{tabular}{|c|c|c|c|}
\hline Colombia & Ecuador & Perú & Bolivia \\
\hline Pendiente & $\begin{array}{l}\text { Distancia a áreas } \\
\text { transformadas }\end{array}$ & $\begin{array}{l}\text { Distancia a } \\
\text { minería }\end{array}$ & $\begin{array}{l}\text { Distancia a áreas } \\
\text { transformadas }\end{array}$ \\
\hline Altitud & $\begin{array}{l}\text { Distancia a } \\
\text { caminos }\end{array}$ & Pendiente & Pendiente \\
\hline $\begin{array}{l}\text { Distancia a } \\
\text { caminos }\end{array}$ & $\begin{array}{l}\text { Distancia a } \\
\text { minería }\end{array}$ & Altitud & Altitud \\
\hline Distancia a ríos & Pendiente & $\begin{array}{l}\text { Distancia a áreas } \\
\text { transformadas }\end{array}$ & $\begin{array}{l}\text { Distancia a } \\
\text { caminos }\end{array}$ \\
\hline $\begin{array}{l}\text { Distancia a áreas } \\
\text { transformadas }\end{array}$ & Altitud & $\begin{array}{l}\text { Distancia a } \\
\text { caminos }\end{array}$ & $\begin{array}{l}\text { Distancia a } \\
\text { pobla-dos } \\
\text { mayores }\end{array}$ \\
\hline $\begin{array}{l}\text { Distancia a } \\
\text { minería }\end{array}$ & $\begin{array}{l}\text { Distancia a } \\
\text { pobla-dos } \\
\text { mayores }\end{array}$ & Distancia a ríos & $\begin{array}{l}\text { Distancia a } \\
\text { minería }\end{array}$ \\
\hline $\begin{array}{l}\text { Distancia a } \\
\text { pobla-dos } \\
\text { mayores }\end{array}$ & Precipitación & $\begin{array}{l}\text { Distancia a } \\
\text { pobla-dos } \\
\text { mayores }\end{array}$ & Distancia a ríos \\
\hline Precipitación & Distancia a ríos & Precipitación & Precipitación \\
\hline
\end{tabular}

Potencial de deforestación al 2030.-Este dato fue calculado mediante la herramienta Land Change Modeler del software de SIG IDRISI Selva que requiere de datos de deforestación reales de series de tiempo y datos sobre las variables potencialmente asociadas a los patrones de deforestación observados (Eastman et al., 2005). Con estos insumos genera un modelo a una fecha determinada por el usuario cuyo resultado representa el potencial relativo de transición o deforestación por pixel ("soft prediction model”).

Como insumos para el modelo se usaron una línea base de cobertura y uso al año 2000 y los datos de deforestación 2004-2009 procesados por Terra-i, un algoritmo que usa los datos de sensores remotos MODIS-NDVI ("Moderate Resolution Imaging Spectroradiometer-Normalized Difference Vegetation Index") y TRMM (“Tropical Rainfall Measuring Mission”) para identificar cambios de cobertura. Los datos representan las detecciones anuales acumuladas de cambio de cobertura del suelo de lo cual se deriva 
información por cada pixel a una resolución de $250 \mathrm{~m}$ (Reymondin et al., 2012). Como variables explicativas se probaron más de 20 variables ambientales incluyendo fuentes de presión y luego de análisis de sensibilidad de las variables independientes y de pruebas de validación del modelo, se seleccionaron las siete variables más potentes para explicar los patrones de deforestación. La validación se hizo a partir de un modelo de deforestación al 2009 generado con los datos entre el 2004 y 2008, de modo que el modelo al 2009 se pudiese validar con los datos reales de deforestación para ese año. Esta validación sirvió para escoger el grupo de variables definitivas para el modelo al 2030 (Tabla 3), así como el ámbito geográfico más apropiado para correr el modelo, que resultó ser por país dentro del área de estudio. Dado que algunas de las variables usadas son variables dinámicas, por ej., distancia a áreas ya transformadas, los modelos se corrieron de forma iterativa en cinco fechas (cada cuatro años entre 2010 y 2030) para incluir en cada fecha las áreas proyectadas de deforestación como nuevos insumos para la estimación de deforestación a años futuros. El resultado de deforestación estimada se calculó directamente por unidad de cuenca según el número de pixeles con potencial de deforestación a 2030 versus el número de pixeles que permanecen con cobertura forestal o natural.

La calificación final de las amenazas combina los tres componentes mencionados utilizando un esquema de ponderación donde cada uno contribuye con 1/3 de la calificación. Antes de combinarlos, los resultados de cada uno de los tres componentes se normalizaron dividiendo por el valor máximo por cuenca de modo que todas las entradas tienen un valor máximo de 1 . El valor combinado de amenazas también se normalizó a una escala de $0-1$.

4 Servicios ecosistémicos.

Potencial para emisiones evitadas de carbono. Un análisis del potencial de una cuenca para proveer el servicio de evitar emisiones de carbono que serviría en el contexto de REDD+ requiere información sobre la distribución de carbono y la tasa de conversión de vegetación natural a otros usos de suelo. El potencial de evitar emisiones de carbono es el producto de la cantidad de carbono almacenado y la tasa de conversión. Se utilizó datos de la distribución de carbono según la biomasa subterránea y sobre el suelo de una base de datos con una resolución de $1 \mathrm{~km}^{2}$ para las regiones tropicales en todo el mundo derivada de datos de sensores remotos, LIDAR y parcelas de inventario forestal (Saatchi et al., 2011). Se calculó el promedio de carbono almacenado (en $\mathrm{Mg} / \mathrm{ha}$ ) para cada cuenca.

Los datos de tasa de deforestación (Terra-i 20042009) indican la pérdida de la cobertura vegetal por cada pixel de 250 x $250 \mathrm{~m}$. Se calculó un promedio de tasa de deforestación anual para cada cuenca y un promedio por pixel en la cuenca (eliminando los pixeles calificados como deforestados según la línea base al año 2000) para calcular la tasa anual de deforestación. Para el valor final del potencial para emisiones evitadas de dióxido de carbono, se multiplicó este resultado por el promedio de carbono almacenado en la cuenca y por un factor de conversión propuesto por Larsen et al. (2011) de $3.66 \mathrm{tCO}_{2} / \mathrm{t} \mathrm{C}$.

Provisión de recursos hídricos. - Se usó la información desarrollada por Larsen et al. (2011) basada en una metodología para estimar el suministro de recursos hídricos a las poblaciones humanas. El cálculo se basó en cuatro componentes: precipitación, flujo hacia cursos de agua conectados, tamaño de la población humana beneficiada y la calidad del agua. Se usó una grilla de hexágonos de $2592 \mathrm{~km}^{2}$ en los cuales se calculó el caudal de agua que se escurre de cada hexágono (Alcamo et al., 2003), el flujo hacia hexágonos adyacentes de elevaciones menores (U.S. Geological Survey, 2000; Lehner, 2008) y la demanda de agua en esos hexágonos según el tamaño de su población humana en 2006. Se usó información sobre la cobertura del suelo en cada hexágono para modificar el cálculo de flujo y para reflejar la calidad del agua. El flujo y calidad del agua es mejor en áreas de bosque, humedales o hielo/nieve (como en los glaciares), intermedio en pastizales naturales y bajo en áreas agrícolas o urbanas (Larsen et al., 2011). Para calcular la provisión de agua de cada cuenca, se calculó el promedio ponderado por área de todos los hexágonos que se traslapan con la cuenca.

Para obtener el valor agregado por cuenca, cada servicio se normalizó primero a una escala entre $0-1$, dividiendo los valores por cuenca para el valor máximo de todas, y luego los valores de los dos servicios fueron promediados y re-normalizados a una escala de $0-1$ para obtener el valor combinado de servicios ecosistémicos por unidad de cuenca.

5 Costos de conservación.

La representación de costo de oportunidad para la conservación combina información de dos fuentes de datos diferentes: información sobre las oportunidades de conservación, recopiladas durante un taller de expertos sobre la región de estudio y datos espaciales sobre la rentabilidad de tierras agrícolas (Naidoo \& Iwamura, 2007).

En mayo del 2012 se llevó a cabo en Lima, Perú, un taller de expertos que reunió a 21 participantes provenientes de organizaciones no gubernamentales de los cuatro países y seleccionados por su experiencia y participación activa en proyectos de conservación y desarrollo sustentable de carácter regional dentro del área del estudio. Los participantes respondieron a un formulario donde se listaron nueve oportunidades y condiciones que facilitarían el éxito de las actividades de conservación (Tabla 4), categorizando el estado de avance de las mismas en el contexto de cada cuenca en cada país, usando para ello el contexto de las 
unidades de cuenca de nivel 4. La calificación estaba restringida a cuatro categorías predeterminadas: escaso, medio, alto y consolidado. Cuencas donde ha habido mayor inversión y actividad en conservación generan una base de experiencia e información, además de la posibilidad de alianzas que representarían mayores oportunidades para emprender acciones de conservación y gestión sustentable. En el marco conceptual utilizado para este estudio, mayores oportunidades de este tipo representan un menor costo para nuevas inversiones en conservación.

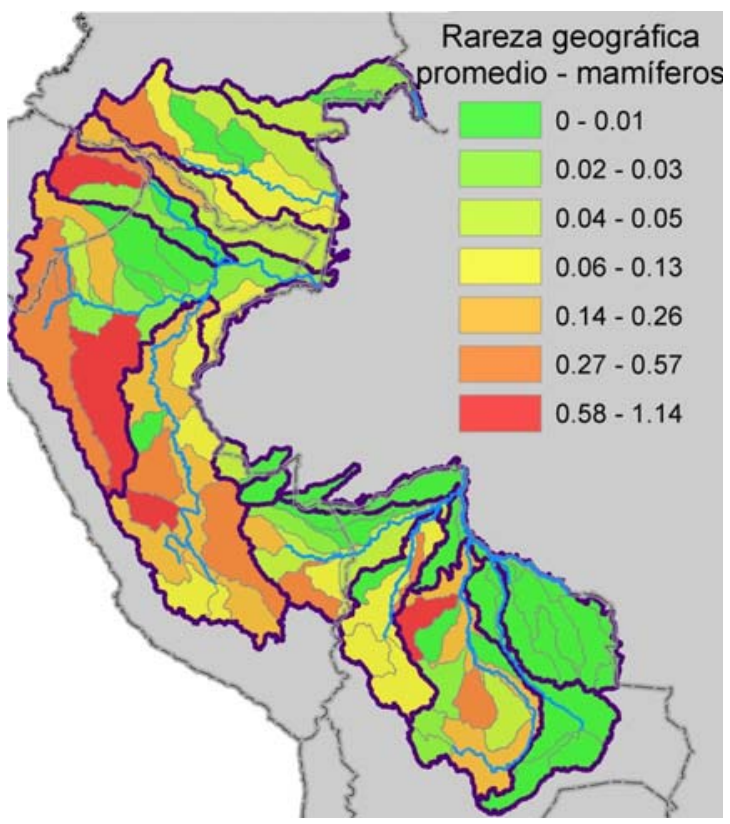

Figura 3. Mapa del área del estudio mostrando la organización de unidades de cuenca adoptada para los análisis y las áreas protegidas.

Para obtener una calificación integrada del estado de las oportunidades, se valoraron las categorías en $0.25,0.50,0.75$ y 1.00 , respectivamente y se sumaron todas las respuestas para cada una de las cuencas. El valor por cuenca se convirtió a una calificación de costo entre 0-1 mediante la división por la máxima calificación de oportunidad de todas las cuencas y utilizando luego el inverso de este resultado; de modo que una calificación más alta representa mayor costo. Este resultado se combinó entonces con el componente de costo de oportunidad, entendido como la renta de actividades agropecuarias que dejaría de obtenerse al dedicar las áreas a actividades de conservación. Para ello la información provino de la publicación de Naidoo \& Iwamura (2007) que reporta datos georeferenciados sobre productividad de 42 tipos de cultivos y de actividades ganaderas combinándolos con precios de productor para estimar la renta bruta por cada unidad de tierra. Los valores por cuenca para cada componente de costo se normalizaron a una escala de $0-1$, se promediaron y re-normalizaron a una escala de $0-1$ para obtener un valor final de costo.

\section{Resultados.}

Animales y plantas. - De los grupos taxonómicos analizados, hay 460 especies listadas en alguna categoría de amenaza, de las cuales un $88 \%$ no cuenta con una cobertura adecuada en los sistemas nacionales de áreas protegidas según las metas establecidas por Rodrigues et al. (2004) (Tabla 5). Los patrones de las

Tabla 4. Lista de condiciones para calificar su estado de avance a nivel de cuenca, utilizado en Taller de Expertos (Lima, Perú, 2012)

\section{Oportunidades y condiciones para el éxito de las} actividades de conservación

Implementación de proyectos de Carbono (esquemas de REDD u otros)

Esquemas de pago por servicios ambientales

Efectividad de la descentralización administrativa para incidir en temas ambientales

Marco legal regulatorio suficiente

Capacidad de las instituciones locales (Dirección

Ambiental, universidad, entes de planificación)

Desarrollo humano (educación, salud, ingreso)

Fortaleza y visión de las organizaciones indígenas

Asignación de recursos económicos para la conservación y manejo de recursos (o inversiones en conservación por diferentes sectores)

Desarrollo e implementación de planes integrales de desarrollo sostenible

cuencas con alta rareza geográfica total promedio son bastante parecidos a través de los tres grupos de vertebrados y las plantas (Figuras 3-6). En general, las cuencas en las zonas altas en el área del estudio demuestran mayores valores de rareza geográfica total que las cuencas en las zonas bajas. En particular, las partes altas de las cuencas del río Marañón en Ecuador y Perú y la parte alta de la cuenca del río Napo en Ecuador son consistentes en tener altos valores de rareza geográfica total promedio tanto para aves, mamíferos y anfibios como para plantas. Cuencas en zonas bajas como en partes de las cuencas del río Caquetá, Alto Purús y Guaporé tienen valores bajos para esta medida. Una excepción es la cuenca del Nanay cerca de Iquitos, Perú, que es importante solamente para aves debido a algunas especies endémicas al bosque de arena blanca ubicado allí.

Mamíferos. - El análisis demuestra que la rareza geográfica total promedio para mamíferos amenazados y no protegidos ( $n=43$ especies) se concentra en las zonas altas a través del área del estudio (Figura 3). La única excepción es en la cuenca del Yacuna de la sabana del Beni en Bolivia donde se encuentran dos especies endémicas de primate (Callicebus modestus y 
C. olallae). Los valores de rareza geográfica total promedio varían entre 0.0 y 1.14 (Figura 3). Estas cifras bajas reflejan que los mamíferos amenazados y no protegidos que habitan el área estudiada tienden a tener distribuciones más amplias que los otros grupos focales (Tabla 5).

Tabla 5. Resumen de los resultados para especies. Ver texto para la definición de 'no protegido'.

\begin{tabular}{|c|c|c|c|}
\hline Grupo & $\begin{array}{c}\text { Número de } \\
\text { especies } \\
\text { amenazadas }\end{array}$ & $\begin{array}{c}\text { Porcentaje } \\
\text { de las } \\
\text { especies } \\
\text { amenazadas } \\
\text { que no son } \\
\text { protegidas }\end{array}$ & $\begin{array}{c}\text { Mediana del } \\
\text { tamaño de la } \\
\text { distribución de las } \\
\text { especies } \\
\text { amenazadas y no } \\
\text { protegidas dentro } \\
\text { del área del } \\
\text { estudio }\left(\mathrm{km}^{2}\right)\end{array}$ \\
\hline Mamíferos & 63 & 68 & 36572 \\
\hline Aves & 100 & 84 & 12255 \\
\hline Anfibios & 206 & 93 & 2905 \\
\hline Plantas (total) & 91 & 95 & 6396 \\
\hline Arecaceae & 21 & 86 & 15411 \\
\hline Brunelliaceae & 14 & 100 & 2140 \\
\hline Cactaceae & 11 & 91 & 7542 \\
\hline Passerifloraceae & 45 & 98 & 3801 \\
\hline Todos & 460 & 88 & 9168 \\
\hline
\end{tabular}

Anfibios. - Los anfibios ( $\mathrm{n}=192$ especies amenazadas y no protegidas) demuestran un patrón parecido al patrón de los mamíferos, con altos valores de rareza geográfica total en las zonas altas (Figura 4). En el caso de los anfibios, las cuencas bajas de los ríos

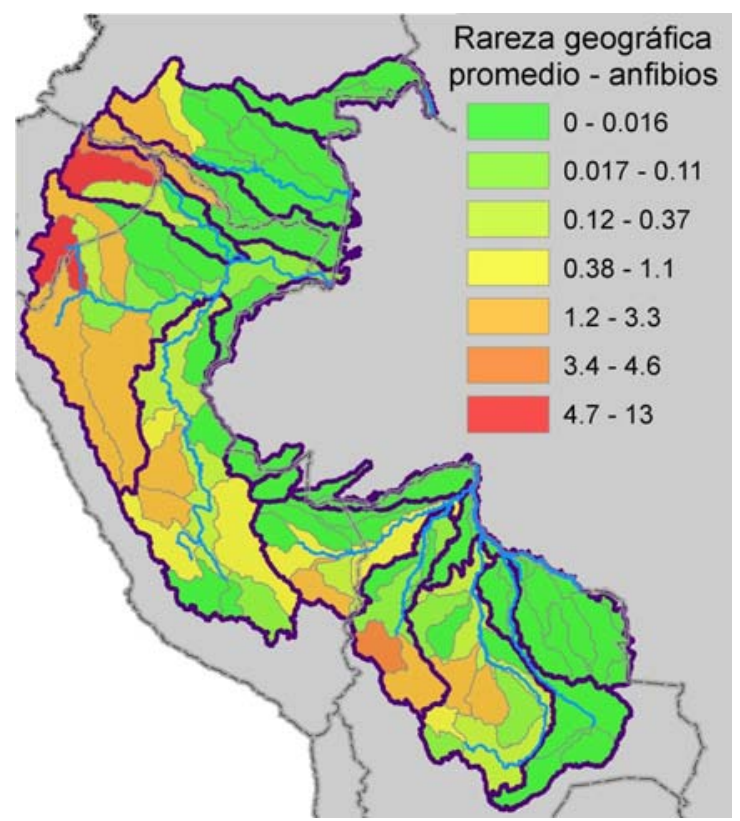

Figura 4. Rareza geográfica promedio de los anfibios amenazados y no protegidos. Valores mayores indican cuencas con más especies de distribuciones muy reducidas y por tanto, con menores opciones de conservación.
Marañón y Ucayali y los ríos colombianos tienen valores aún menores que los valores de los mamíferos. Existe una cuenca de zona baja con un valor intermedio, es la cuenca del río Madre de Dios, río abajo de Puerto Maldonado. En la cordillera andina, hay dos cuencas, Pacobamba y Vilcabamba al oeste y sur de Cuzco centrado en la Cordillera de Vilcabamba, que demuestran valores relativamente bajos en comparación con el resto de la cordillera. Los valores de rareza geográfica total promedio son en general altos en anfibios, alcanzando 13.10 para una cuenca, mucho más alto que para cualquier otro grupo taxonómico. Los anfibios, como grupo, tienen muchas especies amenazadas y de distribuciones reducidas, y los resultados indican que muchas de ellas están fuera de las áreas protegidas.

Aves. - Las aves $(n=84$ especies amenazadas y no protegidas) demuestran un patrón parecido al patrón de los anfibios con altos valores en las zonas altas y bajos valores en las cuencas bajas, con la única excepción para zonas bajas en la cuenca del Nanay cerca de Iquitos mencionada anteriormente (Figura 5). Los valores son levemente menores también en la Cordillera de Vilcabamba, pero además en Ecuador, en la cabeza del río Pastaza y en la alta cuenca del río Putumayo en la zona fronteriza entre Ecuador y Colombia. Los

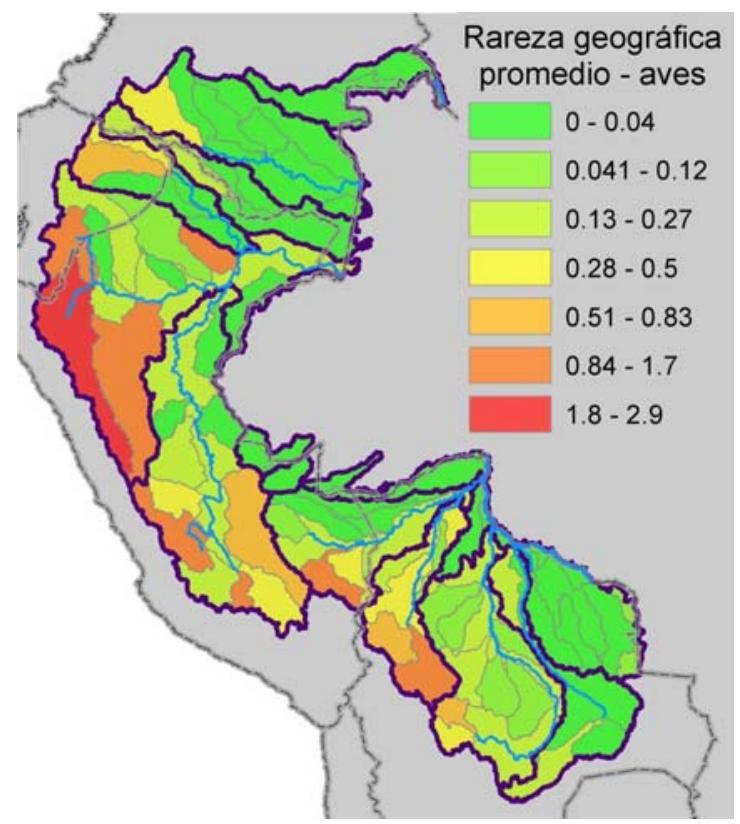

Figura 5. Rareza geográfica promedio de las aves amenazadas y no protegidas. Valores mayores indican cuencas con más especies de distribuciones muy reducidas y por tanto, con menores opciones de conservación. 
valores de rareza geográfica total promedio son relativamente bajos, alcanzando como máximo 2.87 .

Plantas. - Los resultados para las familias de plantas que se pudieron analizar ( $\mathrm{n}=86$ especies amenazadas y no protegidas) de nuevo resaltan el valor de la mayor parte de las cuencas altas en la cordillera andina (Figura 6). Los valores más bajos ocurren en la cuenca del río Vaupés en Colombia y en las cuencas Guaporé y Negro/San Martín en Bolivia. Sin embargo, de todos los grupos analizados, las plantas demuestran mayores valores en cuencas al pie de monte. Esta diferencia se nota más en las subcuencas del Río Marañón en Perú. Además una subcuenca baja, la de Yavarí, también tiene altos valores a diferencia de los tres grupos de vertebrados estudiados. Los resultados para las plantas agregan los patrones de las cuatro familias analizadas. Brevemente, las áreas de alta rareza geográfica de Arecaceae son las cuencas altas de Ecuador hasta Bolivia y de menor importancia, la cuenca del río Marañón. Las Passifloraceae mostraron alta rareza geográfica solamente en las cuencas altas entre el sur de Ecuador hasta Bolivia. Para Brunelliaceae, los valores son más altos también en las cuencas altas. Las Cactaceae mostraron valores altos en cuencas altas e intermedias. Los valores absolutos de rareza geográfica total promedio son intermedios cuando se los suman para las cuatro familias, con el valor más alto de 3.61.

Ecosistemas terrestres. - Los resultados por

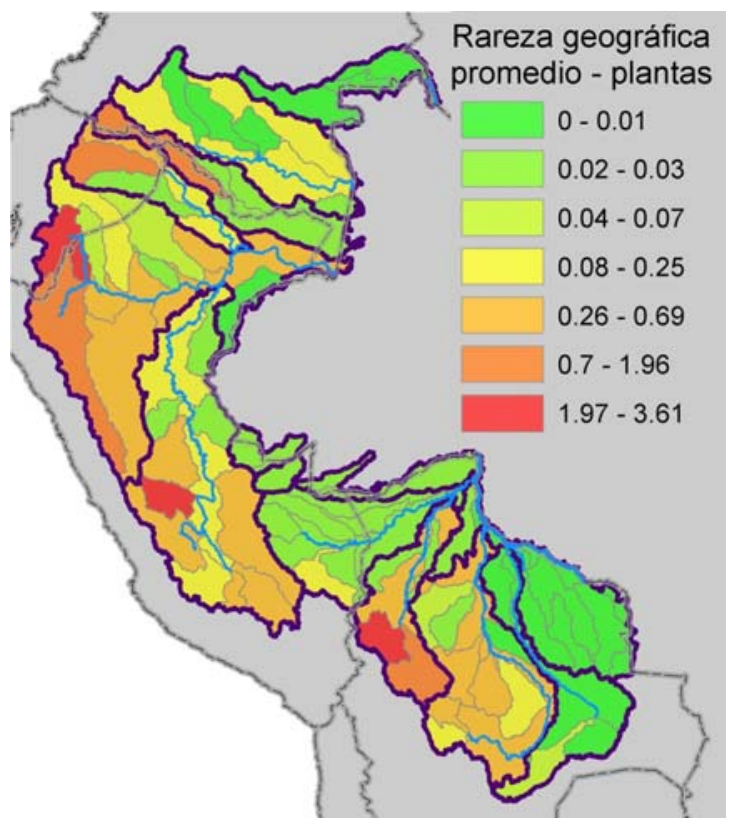

Figura 6. Rareza geográfica total promedio de especies amenazadas y no protegidas de cuatro familias de plantas (Arecaceae, Brunelliaceae, Cactaceae y Passifloraceae).

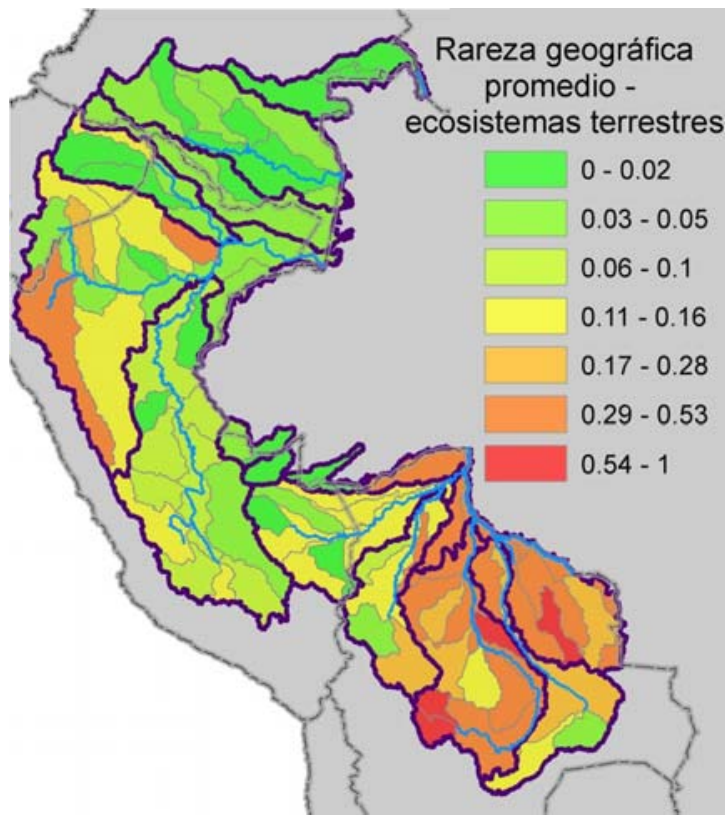

Figura 7. Rareza geográfica de ecosistemas terrestres ponderada con integridad. Valores normalizados entre 0-1. La calificación resalta cuencas con proporciones altas de área en buena condición de ecosistemas muy restringidos en su distribución total e insuficientemente protegidos.

cuenca para los ecosistemas terrestres (Figura 7) resaltan la importancia relativa de cuencas como la del Mamoré y Guaporé en Bolivia, además de las cabeceras de la cuenca del Marañón en Perú. Este resultado se debe a la presencia de una alta diversidad de ecosistemas en áreas de ecotono entre diferentes regiones biogeográficas como son la Amazonía, el Beni y la Chiquitania en Bolivia y, además, a una alta diversidad de hábitats gracias a las características físicas y ambientales de estas áreas, como por ejemplo, valles interandinos xéricos y extensas llanuras de inundación estacional. Cabe resaltar que tanto la cuenca del Mamoré como las cabeceras del Marañón sobresalen por sus altos valores de rareza geográfica de ecosistemas pese a tener valores de condición del paisaje relativamente bajos, lo que indica que estas cuencas tienen una alta representatividad de ecosistemas de distribución restringida, que hace que sobresalgan pese a la posible disminución de su integridad ecológica. Otro aspecto que influye en este resultado es la escasa representación en las áreas protegidas de ecosistemas como los de las sabanas del Beni o las jalcas y bosques y matorrales xéricos de los valles interandinos del alto Marañón, vacíos identificados ya por Swenson et al. (2012).

Ecosistemas acuáticos. - Al comparar el mapa de diversidad de ecosistemas acuáticos por cuenca con el de rareza geográfica ponderada (Figuras 8 y 9), los 
resultados indican que valores bajos de condición eliminan a cuencas ricas en ecosistemas acuáticos no protegidos (Alto Marañón, Huallaga, Ucayali, Madre de Dios y Mamoré), y favorecen la calificación final de cuencas de riqueza intermedia pero con mejores condiciones de integridad y conectividad, como es el caso de buena parte de la Amazonía colombiana, Masisea en Ucayali, Huáscar y Manu en Madre de Dios, Parapetí e Iténez en Bolivia. Resultados que no conforman con esta explicación, corresponden a la expresión directa del nivel de rareza geográfica. Todo esto hace que las cuencas más importantes para la biodiversidad acuática presenten una distribución bastante dispersa.

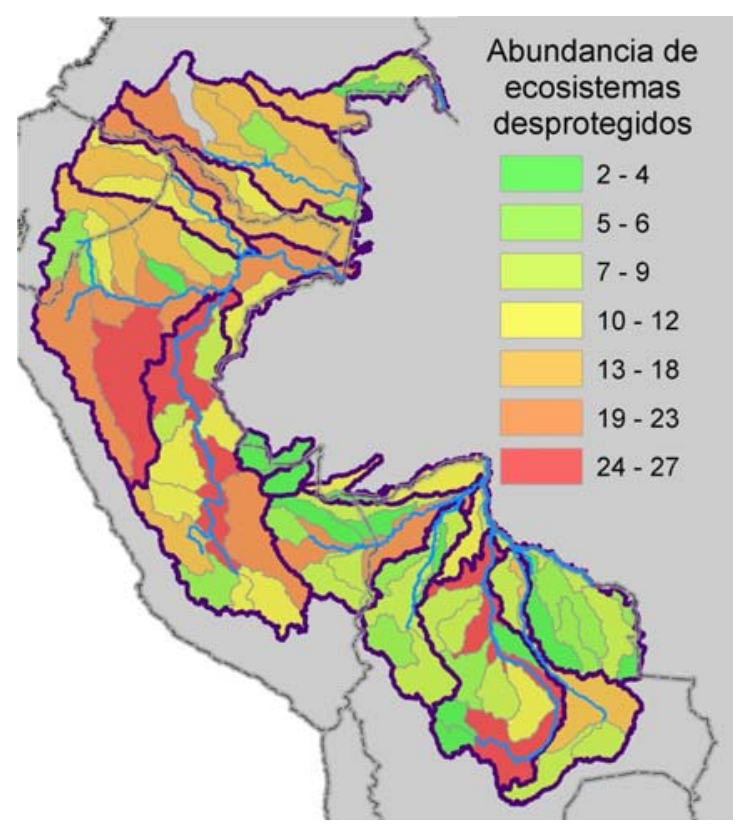

Figura 8. Abundancia de ecosistemas acuáticos insuficientemente protegidos por cuenca.

Amenazas. - Los tres factores que se consideraron para el análisis de amenazas tienen una expresión espacial distinta. En la condición del paisaje, las carreteras y concesiones mineras a futuro no suponen mayores cambios a la condición ya afectada de las cabeceras de cuenca en general y de la cuenca del Mamoré (Figura 10a), mientras tanto es llamativo el deterioro a futuro de las cuencas media y baja del Marañón, Ucayali y Madre de Dios (Figura 10b). En la conectividad hidrológica el deterioro es relativamente confinado; es decir, que si bien el impacto se intensifica a futuro, no se expande a nuevas subcuencas salvo excepciones en las subcuencas del Ucayali y en Inambari (Figura $11 \mathrm{a}, \mathrm{b}$ ). Esto indica una alta concentración en la localización de las 59 represas planificadas.

La proyección de las amenazas en su conjunto se complementa con el uso del potencial de deforestación (Figura 12), donde se añaden otras variables directas o

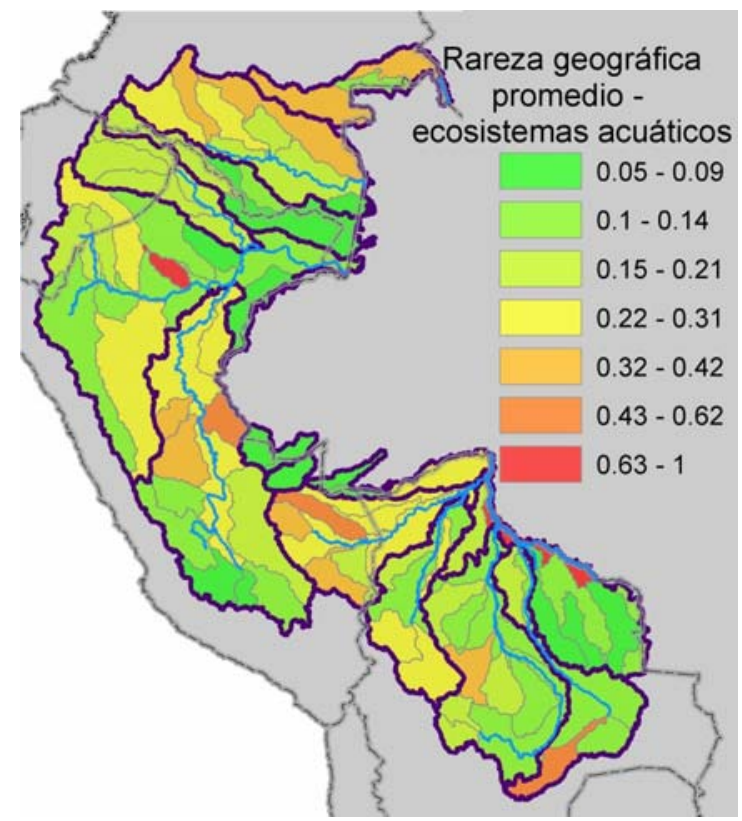

Figura 9. Rareza geográfica de ecosistemas acuáticos ponderada con integridad. Valores normalizados entre 0-1. La calificación resalta cuencas con proporciones altas de área en buena condición de ecosistemas muy restringidos en su distribución total e insuficientemente protegidos.

sucedáneas, que tienen correlación con las tendencias observadas de deforestación. La deforestación estimada a 20 años muestra mayor concentración en las cuencas bajas, es decir hacia el límite oriental del área de estudio, aunque hay valores altos en las cabeceras de cuenca del Napo y Marañón en Ecuador. Una vez que este factor se integra al análisis, la distribución de las amenazas es más generalizada y alcanza a Colombia, que en los otros dos factores prácticamente no muestra impactos, esto debido a que la cuenca alta del Putumayo y Caquetá tienen también puntajes altos de potencial de deforestación.

\section{Servicios ecosistémicos}

Potencial para emisiones evitadas de carbono. El almacenaje de biomasa de carbono es mayor en los bosques lluviosos de zonas bajas donde los árboles tienden a ser altos y de mucha biomasa (Figura 13a). Cuencas de zonas altas, con pastizales como el páramo o la puna, o con bosque andino, junto con cuencas de áreas de sabana como en el Beni de Bolivia almacenan menos biomasa de carbono. Tomando en cuenta toda el área del estudio, los promedios varían entre 11.5 $\mathrm{Mg} / \mathrm{ha}$ en la cuenca del Río Caine, Bolivia, a 194.5 Mg/ha en la cuenca del Río Purús, Perú. La tasa de deforestación varió de casi nulo (cuencas de los ríos Caine, Tucavaca y San Pedro) hasta $0.59 \%$ por año (Figura 13b). Las cuencas con la mayor tasa de deforestación en el periodo estudiado (2004-2009) son Caguán y Yari en Colombia, Manuripi entre Perú y 
Bolivia y Mamoré y Yapacaní en Bolivia. Cuando se multiplicó las cifras de biomasa de carbono con las tasas observadas para obtener el potencial para evitar emisiones de dióxido de carbono, las mismas cuencas

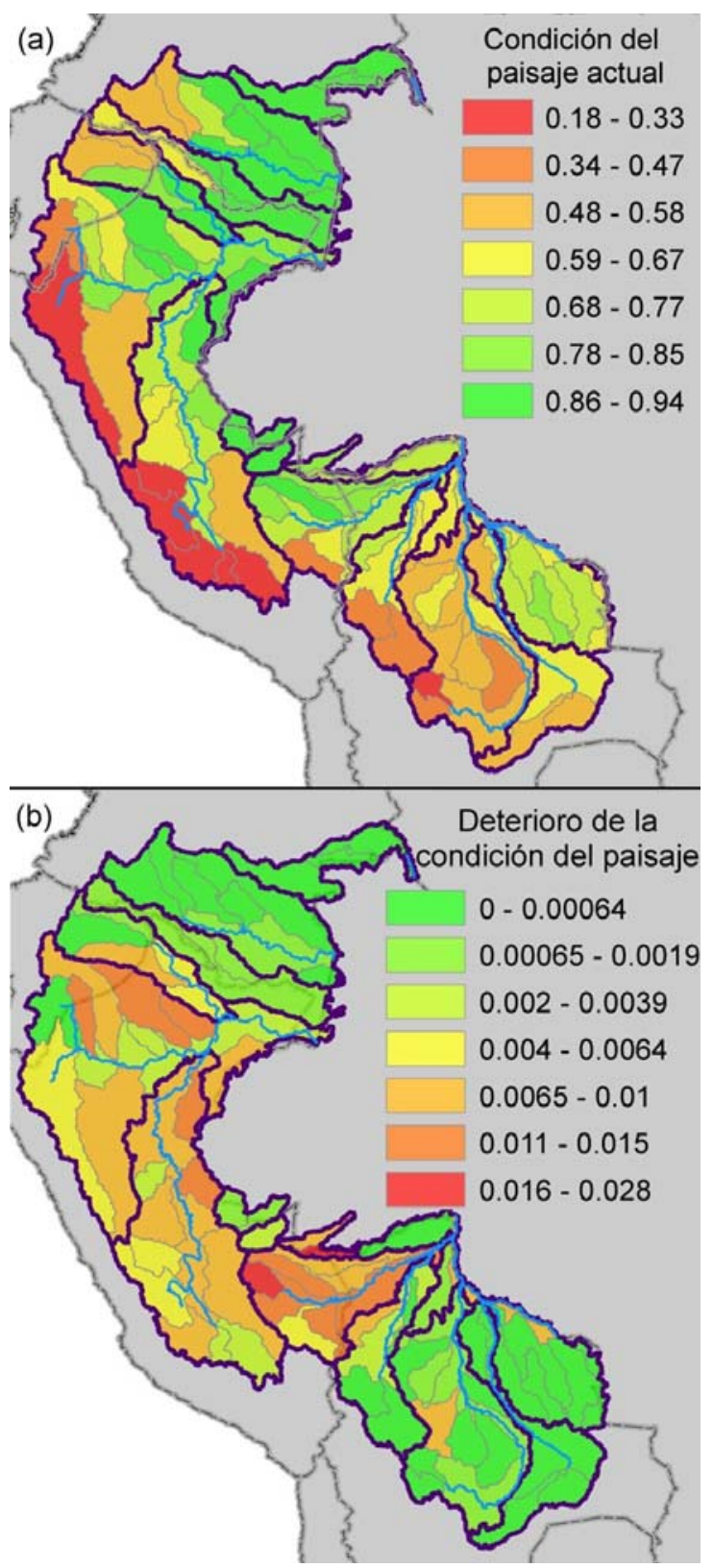

Figura 10. a) Condición del paisaje actual. El valor es un índice del impacto acumulado sobre la condición del paisaje por los diferentes usos del suelo y la presencia de infraestructura, $0=$ mala condición y 1= excelente condición.

b) Deterioro estimado de la condición del paisaje a futuro. Valores mayores indican cuencas con más deterioro proyectado. Para el escenario futuro se usó las carreteras proyectadas y áreas mineras disponibles. con tasas altas de deforestación resaltan como las áreas con más opciones para realizar este servicio (Figura 13c). A estas áreas se agrega la cuenca del río Abuná y varias subcuencas del Río Guaporé en Bolivia donde también hay una tasa bastante alta de deforestación. Casi todas las cuencas de las zonas altas y de pie de montaña tuvieron cifras bajas para el potencial de evitar emisiones de carbono.

Provisión de recursos hídricos. - El análisis resaltó la importancia de las cabeceras de cuencas en el servicio de provisión de recursos hídricos (Figura 14). Este resultado se deriva de varios factores. El factor más importante es la ubicación de grandes centros poblados en esas mismas cuencas, lo que crea una demanda para el agua e incrementa el nivel de aprovisionamiento. Otros factores que influyen son la alta precipitación en zonas altas y especialmente en alturas intermedias y las grandes extensiones de cobertura de vegetación natural que aún permanecen en las cabeceras y que aumenta la calidad del agua provista por estas cuencas.

Costo de las acciones de conservación. Tomando en cuenta exclusivamente el avance de las condiciones habilitantes para la conservación, que se pueden agrupar en: impacto de inversiones en conservación, desarrollo humano e institucional local y marco legal y administrativo vigente, las cuencas con mayores oportunidades son Caquetá en Colombia, Napo y Marañón en Ecuador, Marañón medio y bajo y Madre de Dios en Perú y Mamoré en Bolivia (Figura 15). La inclusión de las rentas anuales de las actividades agropecuarias, con mayores costos de oportunidad ubicados en las cuencas altas (Figura 16), desplaza las oportunidades de conservación hacia las tierras bajas donde los costos para la conservación son menos elevados. La excepción a este patrón es en Bolivia, donde hay costos de oportunidad altos en las áreas de sabana y las dedicadas a la ganadería y soya a partir de la conversión del bosque Chiquitano (Figura 16).

\section{Discusión.}

Animales y plantas. - Los resultados del análisis de cuencas con mayores valores de rareza geográfica total promedio para especies amenazadas y no protegidas resaltan la importancia de las cuencas altas. En todos los grupos estudiados, la gran mayoría de las cuencas más altas en la cordillera andina son las cuencas con valores más altos. Este resultado quizás no es inesperado. Varios estudios han resaltado la importancia de las zonas relativamente altas en la vertiente oriental de la Cordillera de los Andes para especies endémicas (Stattersfield et al., 1998; Duellman, 1999; Knapp, 2002; Van der Werff \& Consiglio, 2004; Fjeldså, 2005; Young et al., 2009; Swenson et al., 2012). Los grupos taxonómicos varían con el gradiente altitudinal, habiendo más concentración de especies endémicas entre 
elevaciones medias y altas (pero siempre por debajo de los ecosistemas no boscosos de muy alta montaña como puna y páramo; Swenson et al., 2012). Sin embargo, a la escala de cuenca que se analizó en este estudio las cuencas de las zonas más altas abarcan tanto las elevaciones medias como las elevaciones altas y así captan la mayoría de las distribuciones de
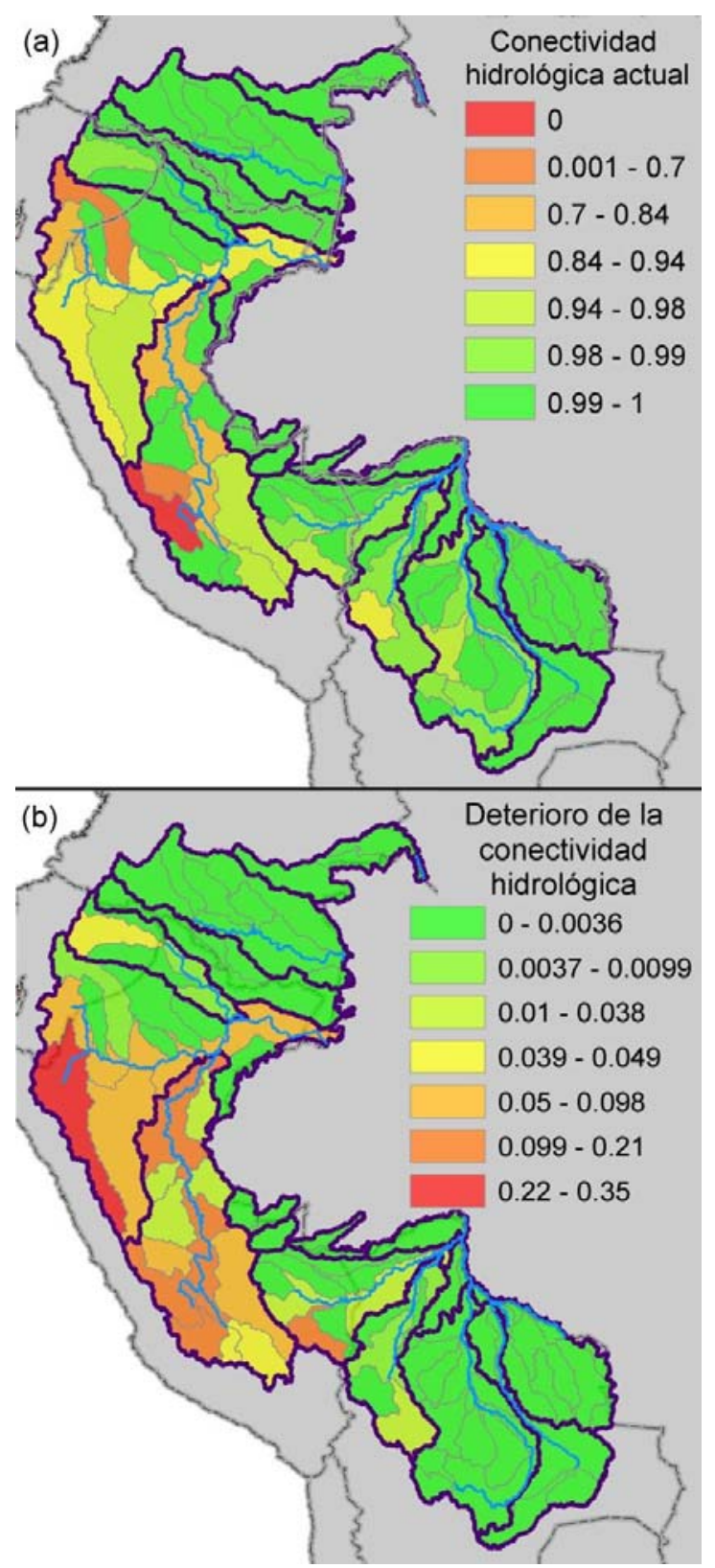

Figura 11. Conectividad hidrológica. a) Estado actual. b) Deterioro estimado de la conectividad hidrológica a futuro. Para estimar el escenario futuro, se incluyeron en el análisis las represas planificadas más probables (Finer \& Jenkins, 2012), que suman 59 para toda el área. las especies endémicas.

En parte los resultados se derivan del hecho de que algunas de las áreas protegidas más grandes están ubicadas en tierras bajas (Figura 2). Su existencia significa que las especies amenazadas de zonas bajas tienden a tener partes de su distribución protegida y así no entran en el análisis por no ser especies no protegidas.

Por lo menos en Ecuador, Perú y Bolivia, se ha notado anteriormente que las distribuciones de muchas especies endémicas no coinciden bien con las ubicaciones de las áreas protegidas (Valencia et al., 2000; Young et al., 2009; Swenson et al., 2012). Nuestro análisis usó una metodología levemente distinta al enfocarse solamente en especies en algún estado de amenaza y no propiamente en especies endémicas a un país o región. El uso de umbrales de tamaño de la distribución en algunos de los criterios de la UICN (UICN, 2001) significa que las especies endémicas serían más propensas a ser evaluadas como amenazadas. En verdad, varios estudios han demostrado una relación negativa entre tamaño de la distribución y grado de amenaza (Owens \& Bennett, 2000; Pitman \& Jørgensen, 2002; Gaston, 2003). Estos resultados hacen hincapié en la observación que aunque se ha avanzado mucho en la creación y manejo de las áreas protegidas en la región, todavía quedan retos importantes para asegurar la conservación de muchas especies únicas.

Se ha argumentado que para el caso de muchas especies endémicas, se necesitan sistemas de reservas pequeñas además de parques grandes "banderas" (Young et al., 2009). La falta de recursos para la conservación y la necesidad de hacer actividades productivas en muchas áreas significa que sería imposible proteger las distribuciones de todas las especies endémicas con parques nacionales. La restricción de algunas especies a pequeñas áreas aisladas sugiere que reservas al nivel de departamento o municipio pueden complementar para llenar los vacíos en la protección de las especies. Además el desarrollo de actividades económicas compatibles con la protección de estas especies es otra alternativa en estos casos. Por ejemplo, la protección de franjas de bosque a los lados de los arroyos en zonas productivas puede ser compatible con la protección a una rana.

Un factor limitante en los análisis de biodiversidad es el nivel de conocimiento. Por ejemplo, hay 346 especies de anfibios de Colombia, Ecuador, Perú y Bolivia evaluadas actualmente como Datos Insuficientes por la falta de información sobre sus tendencias y tamaños poblacionales y sus distribuciones (_revisado 26 de mayo de 2012). Más investigación podría indicar que muchas de estas especies son amenazadas y por lo tanto merecen ser tomadas en cuenta. Por otro lado, hay muchas especies que aún no han sido descubiertas y descritas. Las tasas de descripción de los mamíferos y los anfibios son 
más altas en la actualidad que en cualquier otro momento en la historia de la exploración científica (Köhler et al., 2005; Reeder et al., 2007). Algunas cuencas donde el acceso es muy difícil podrían albergar más rareza geográfica de especies de lo que se aprecia en la actualidad.

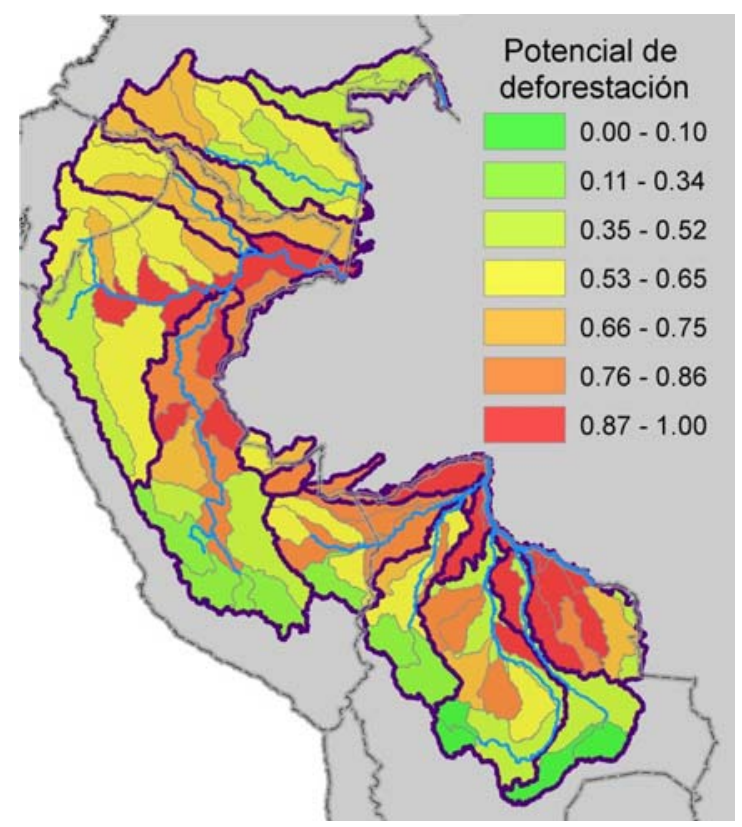

Figura 12. Potencial de deforestación al 2030. Se modeló con Land Change Modeler del software IDRISI Selva usando datos de deforestación de 2004-2009 procesados por Terra-i.

La selección de familias de plantas estudiadas puede ser no muy representativa de la gran diversidad botánica en el área del estudio, en particular los bosques lluviosos de tierras bajas. A pesar de que los criterios para su inclusión no incluyeron la representatividad, resultó que se obtuvieron una variedad de grupos de elevaciones, ecosistemas y hábitos distintos. Las Arecaceae tienen arquitecturas variadas y habitan desde el sotobosque hasta el dosel en bosques de zonas bajas tanto como altas. Las Brunelliaceae son arbustos y árboles de bosques montano bajos a altos. Aunque la diversidad de Cactaceae no es grande en la región, varias especies habitan los valles interandinos xéricos y hay una variedad de formas terrestres y epifitas. Las Passifloraceae son principalmente bejucos de elevaciones bajas e intermedias. Esta variedad y complementariedad de forma y hábitat entre las familias estudiadas es fortuita cuando se considera que no existen datos comprensivos para ninguna otra familia de plantas en nuestra área de estudio.

Ecosistemas terrestres y acuáticos. - De los 221 ecosistemas terrestres del área de estudio, 148 calificaron como no protegidos y se incluyeron en los análisis de rareza geográfica. El patrón de importancia

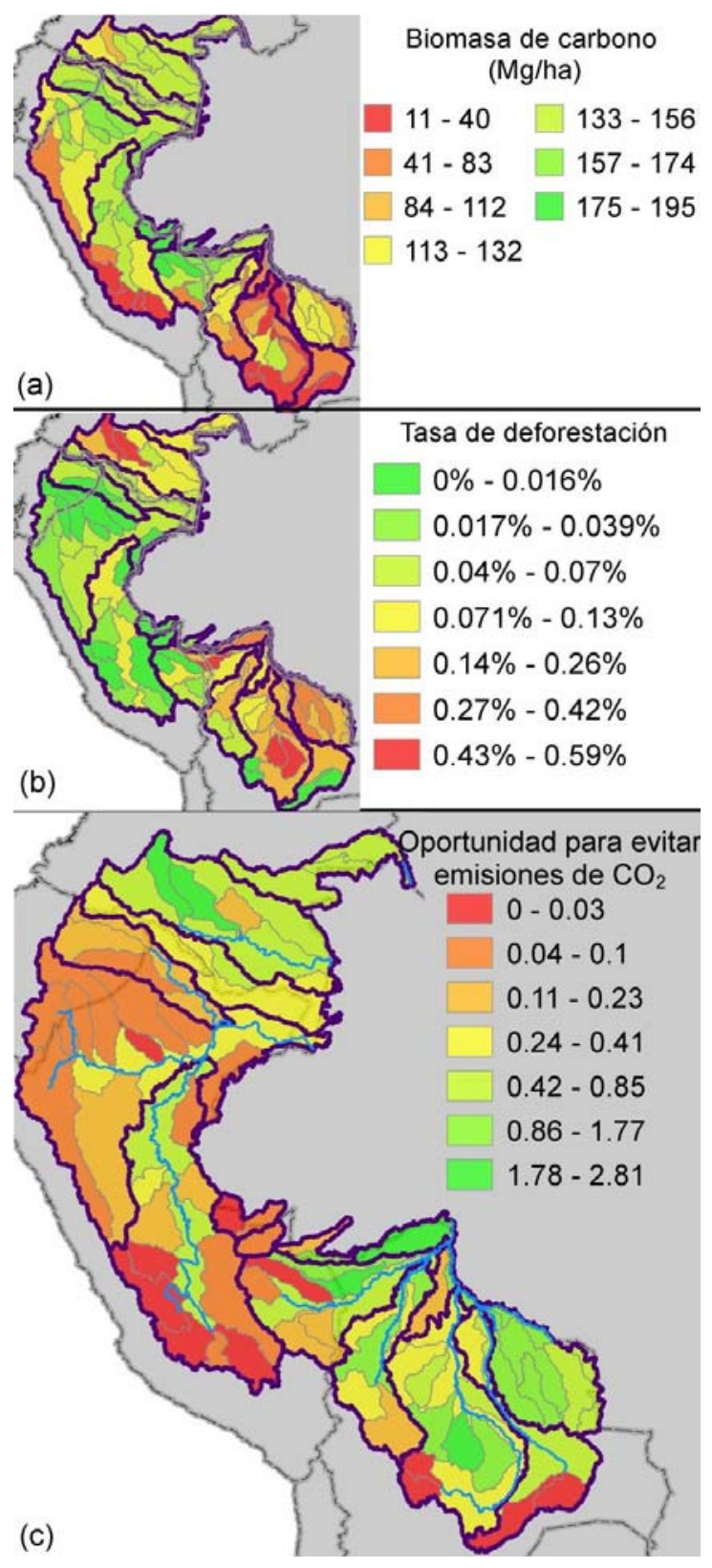

Figura 13. a) Almacenaje de biomasa de carbono (según datos de Saatchi et al., 2011). b) Tasa de deforestación (2004-2009). c) Oportunidad para evitar emisiones de dióxido de carbono (biomasa de carbono multiplicada por tasa de deforestación).

de las cuencas para los ecosistemas refleja claramente la distribución restringida y la falta de protección de los bosques secos y matorrales interandinos xéricos que hace que cuencas como la del Alto Marañón, Vilcabamba y otras en el alto Ucayali en Perú, tengan altos valores (Figura 7). En algunas cuencas, los valores altos de rareza geográfica coinciden con los de 
las especies, principalmente en el caso de las cabeceras del Marañón. En los ecosistemas terrestres se observa un efecto similar al anotado para los ecosistemas acuáticos, donde valores bajos de condición reducen la calificación de cuencas ricas en ecosistemas no protegidos, como la del Ucayali. Por lo demás, algunas de las cuencas de mayor importancia para la protección de ecosistemas terrestres muestran mayores riesgos de amenaza, ya sea por la presencia actual o proyectada de represas y concesiones mineras (Morona, Alto Marañón, Huallaga) (Figura 10 a, b) o debido a la deforestación estimada a futuro en el caso de Nanay en Perú y Abuná, Yata, Iténez, río Blanco y río Negro en Bolivia (Figura 11).

Para los ecosistemas acuáticos, el patrón de importancia de las cuencas es contrastante con el de los ecosistemas terrestres, pues la mayoría de las cuencas prioritarias se encuentran en las tierras bajas y sobre todo con un desplazamiento importante de las prioridades hacia cuencas de la Amazonía colombiana, entre ellas Apaporis y Guainía, y cuencas medias del Ucayali y Madre de Dios en Perú. Si bien para este análisis de rareza geográfica se tomó en cuenta el estado de la conectividad hidrológica en base a las represas existentes, este índice no refleja un impacto muy extendido aún, de manera que el resultado para este factor muestra directamente la unicidad de los ecosistemas considerados. A la vez, denota la falta de protección adecuada de estos ecosistemas únicos.

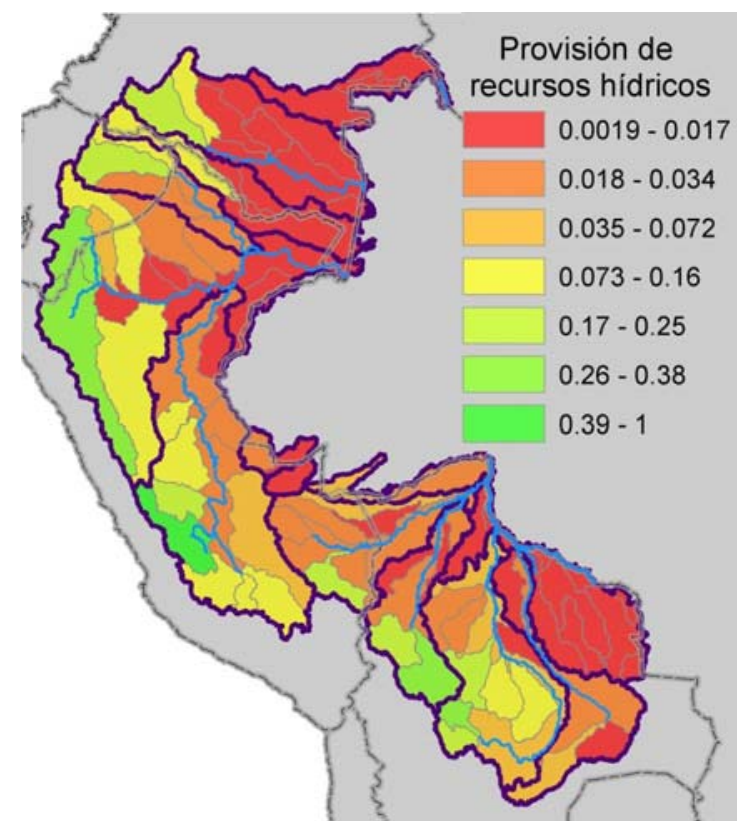

Figura 14. Provisión de recursos hídricos. Valores normalizados entre $0-1$. Los insumos son: precipitación, flujo hacia cursos de agua conectados, tamaño de la población humana beneficiada y la calidad del agua.

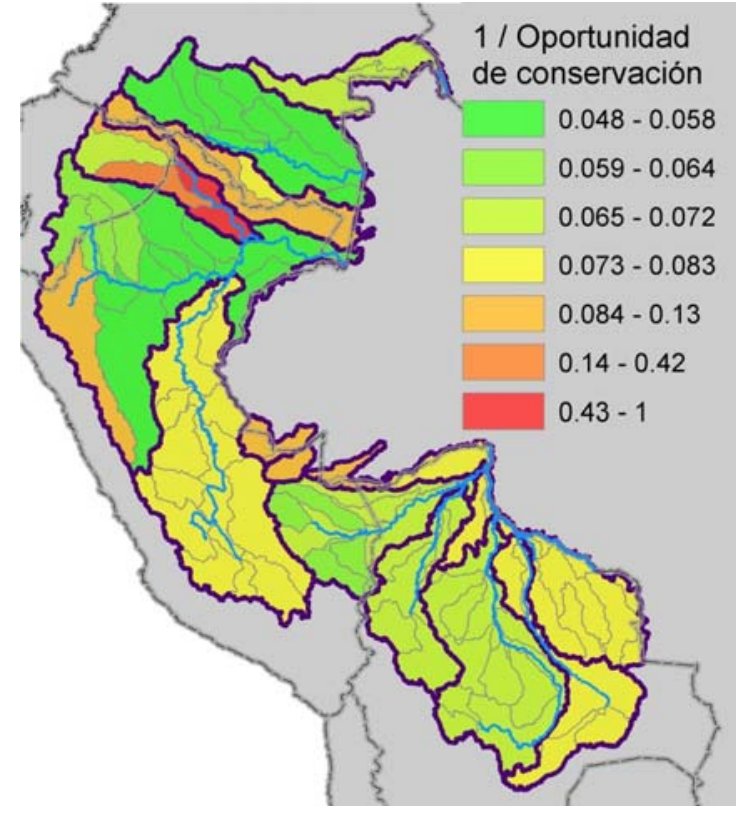

Figura 15. Oportunidad de conservación por el desarrollo de condiciones habilitantes (según opinión de expertos). El color verde indica mayores oportunidades. Valores normalizados entre $0-1$.

Amenazas.- El enfoque utilizado para las amenazas se basó en una estimación del incremento proyectado a futuro de las presiones sobre los elementos de la biodiversidad. Las especies incluidas en el estudio están ya categorizadas como vulnerables, pero este cálculo de las amenazas proporciona una fuente para considerar la vulnerabilidad de todos los elementos de la biodiversidad a diferentes escalas de paisaje, desde cuencas hasta subcuencas menores.

La estimación del nivel de amenaza se ha usado de diferentes maneras en contextos de planificación. Una es para identificar las amenazas que enfrentan los elementos objeto de conservación y definir una estrategia consecuente (The Nature Conservancy, 2007). Con la publicación del concepto y método de la planificación sistemática de la conservación (Margules \& Pressey, 2000) y la difusión de herramientas de apoyo a la planificación, el manejo de la información sobre las amenazas a escala de paisaje se ha utilizado para identificar la manera más eficiente de alcanzar metas de conservación, evitando en lo posible áreas de alto nivel de amenaza en la selección de las áreas de protección o manejo. Sin embargo, un enfoque alternativo para el uso de esta información, al combinarla con el concepto de rareza geográfica de los elementos vulnerables de biodiversidad, es que las áreas más amenazadas son más prioritarias cuando coinciden con valores altos de biodiversidad. Particularmente, cuando el enfoque del análisis de 


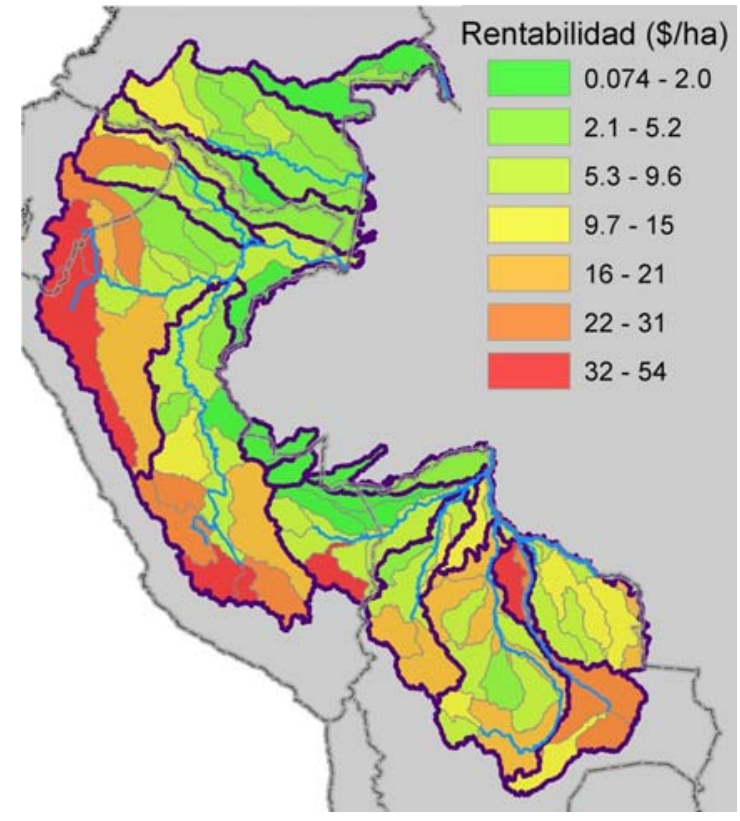

Figura 16. Costo de oportunidad según la rentabilidad potencial de actividades agrícolas (según Naidoo \& Iwamura, 2007).

amenazas es predictivo. Esta aproximación es un ejemplo de la ventaja de desarrollar información dentro del marco lógico presión-estado-respuestabeneficio. Identificar las presiones que potencialmente afectarían a elementos claves de biodiversidad, permite definir respuestas a través de conservación y políticas preventivas.

Se reconoce que la estimación de amenazas a futuro se basa en modelos y en datos susceptibles de errores, sin embargo se aplicaron criterios rigurosos para la inclusión de datos sobre infraestructura planificada, y el modelo de deforestación sobrepasa la calificación aceptable de precisión. Por ello se consideró que el resultado obtenido ofrece una buena representación de los patrones esperables.

Servicios. - La deforestación de las zonas tropicales del mundo contribuye un $20 \%$ a la emisión de dióxido de carbono al nivel global (Houghton, 2005) y por lo tanto hay mucho interés en formular incentivos para reducir la destrucción de bosques tropicales. Nuestros resultados demuestran la gran variedad al nivel de cuenca en la oportunidad de evitar emisiones de estos gases de invernadero. Si se aprueba algún incentivo de REDD, muy probablemente funcionará al nivel de país para evitar "fugas" de deforestación de una región a otra (Ebeling \& Yasué, 2008). Los resultados sirven tanto para identificar zonas donde un proyecto podría tener un importante impacto para evitar muchas emisiones en un país, como para establecer una estimación de una línea base

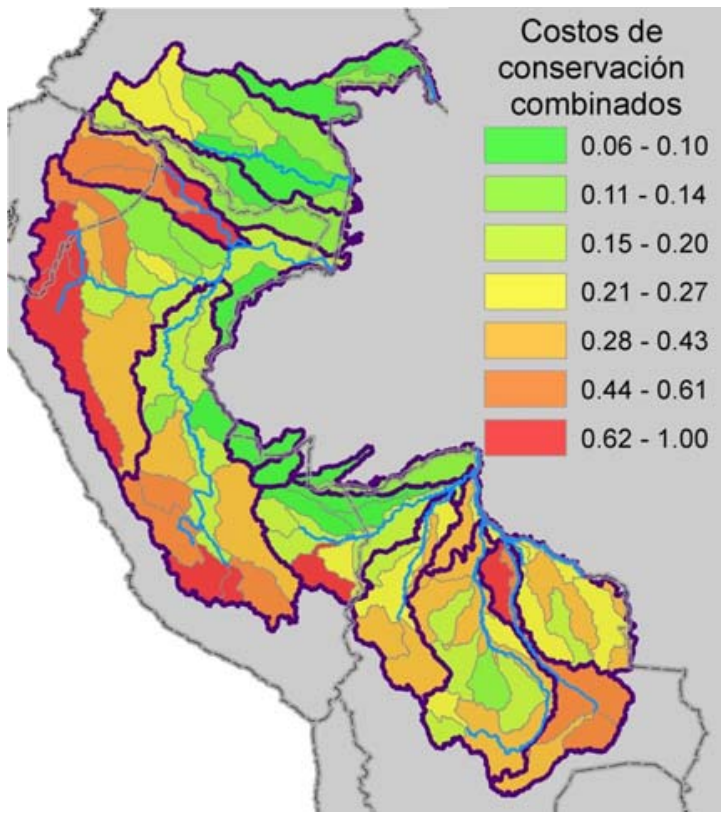

Figura 17. Costos de conservación combinados. Valores normalizados entre 0-1. Representa el promedio de dos componentes: el desarrollo de condiciones sociopolíticas para la conservación y el costo de oportunidad de la renta agrícola potencial.

actual de emisiones causadas por la destrucción de hábitat.

La descripción de la distribución espacial de biomasa de carbono en los trópicos es un área activa de investigación (Le Quéré et al., 2009; Friedlingstein et al., 2010; Saatchi et al., 2011; Baccini et al., 2012). Con la acumulación de datos de miles de parcelas medidas en el campo $\mathrm{y}$ el uso de nuevos $\mathrm{y}$ potencialmente más precisos sensores como el LIDAR, la precisión de las estimaciones ha mejorado sustancialmente en los últimos años. Para este estudio se utilizó la más reciente fuente de datos que cubre por completo nuestro área de estudio (Saatchi et al., 2011). Existe por lo menos una estimación aún más reciente (Baccini et al., 2012) pero no cubre por completo el área del estudio. Los datos de Baccini et al. (2012) sugieren que el análisis de Saatchi et al. (2011) puede haber subestimado la biomasa de carbono en algunas áreas. Si este sesgo aplica igualmente a través del área del estudio, los patrones gruesos que se han demostrado (Figura 13c) no cambiarían.

El área del estudio es la fuente de gran parte de las aguas que fluyen por el río Amazonas, el río con el mayor caudal y la cuenca más grande del mundo. Las cuencas altas en el área del estudio aún cuentan con suficiente cobertura vegetal natural como para proveer agua a unos centros poblados importantes como 
Mocoa (Colombia), Tena (Ecuador), Cusco (Perú) y Cochabamba en Bolivia. Las cuencas de las zonas bajas también contienen caudales importantes, pero debido a la poca población humana se califican con valores menores.

Costos de conservación. - Los elementos incluidos en nuestro análisis identifican las cuencas donde los costos de oportunidad son menores y las condiciones socio-políticas locales relevantes favorecerían a los esfuerzos de conservación. Las cuencas con menores costos se ubican hacia los límites orientales, es decir las cuencas bajas, este es el caso en Colombia y en el Perú, Manuripi y Abuná en Bolivia (Figura 17). Este resultado refleja principalmente los bajos costos de oportunidad en estas áreas, pues cuencas con alta calificación para las condiciones habilitantes pero con costos altos de oportunidad, como las cabeceras y cuenca media del Marañón, no sobresalen en la calificación combinada (Figura 16). Algunos esfuerzos de planificación han utilizado la información sobre las amenazas como un sucedáneo de costos de conservación, asumiendo que remover o mitigar los factores de impacto implica un costo importante. De hecho, estudios realizados en las pampas Argentinas con datos sobre el costo comercial de la tierra y con un análisis de amenazas basado en la densidad de población, tasas de conversión e infraestructura de acceso, han encontrado una alta correlación positiva entre ambos (Murdoch et al., 2010). Sin embargo, nuestro estudio, al utilizar insumos distintos para representar amenazas y costos, muestra que la distribución de ambos es inversa sobre todo en las tierras bajas del área, con costos relativos de conservación bajos en sitios con un riesgo alto estimado a futuro, como ejemplo están la cuenca baja del Marañón, Ucayali y Madre de Dios, además de las cuencas de Juruá y Alto Purús (Figuras 17 y 18). El caso inverso, con costos de conservación altos y amenazas estimadas bajas, se da en la cuenca de Río Negro/San Martín en Bolivia. Este último es un resultado a validar, puesto que el costo de oportunidad de algunas de estas subcuencas no es tan alto como sugieren los datos de rentabilidad agropecuaria.

\section{Conclusiones.}

Con la información generada, este estudio facilita los insumos para una serie de aplicaciones en el marco de la planificación y monitoreo de conservación. Por un lado, mediante las unidades de análisis de cuencas bajo una estructura anidada, se identifican sub-cuencas de mayor importancia relativa dentro de cuencas mayores que, a su vez, sobresalen para los diferentes componentes analizados, lo que tiene relevancia para las actividades y decisiones de conservación al nivel local. La utilización de diferentes componentes de la biodiversidad ayuda a cubrir un rango amplio de elementos con patrones de distribución distintos aunque siempre con un enfoque en los elementos más vulnerables.

Los análisis de amenazas que incluyen tanto un escenario actual como uno futuro para el estado de diferentes factores de presión, es otro aporte de este estudio porque permite una planificación proactiva. En el tema de costo, los factores incluidos en el análisis son relevantes para tomar decisiones al planificar inversiones en conservación; sin embargo, es posible que la resolución espacial de la información aportada por los expertos sobre las oportunidades no refleje diferencias más localizadas. O que, por la edad de los datos sobre la rentabilidad/ha de las actividades agropecuarias, éstos omitan cambios recientes en la accesibilidad, aumentando el valor de tierras consideradas inaccesibles hasta hace poco. En cuanto a los beneficios provenientes de los servicios ecosistémicos, este análisis aporta las calificaciones por unidades de cuenca, lo que facilita comparaciones para la misma unidad entre beneficio y costo, o servicios y biodiversidad.

En definitiva, este estudio pone a disposición pública una serie de capas de información temática que constituyen insumos clave para apoyar la toma de decisiones en cuanto a inversiones en la conservación y para monitorear su impacto en el largo plazo. Se espera que en el futuro se pueda desarrollar los insumos para este tipo de estudio a una escala más fina para así dar apoyo más detallado para la toma de decisiones. Los datos raster que provienen de sensores

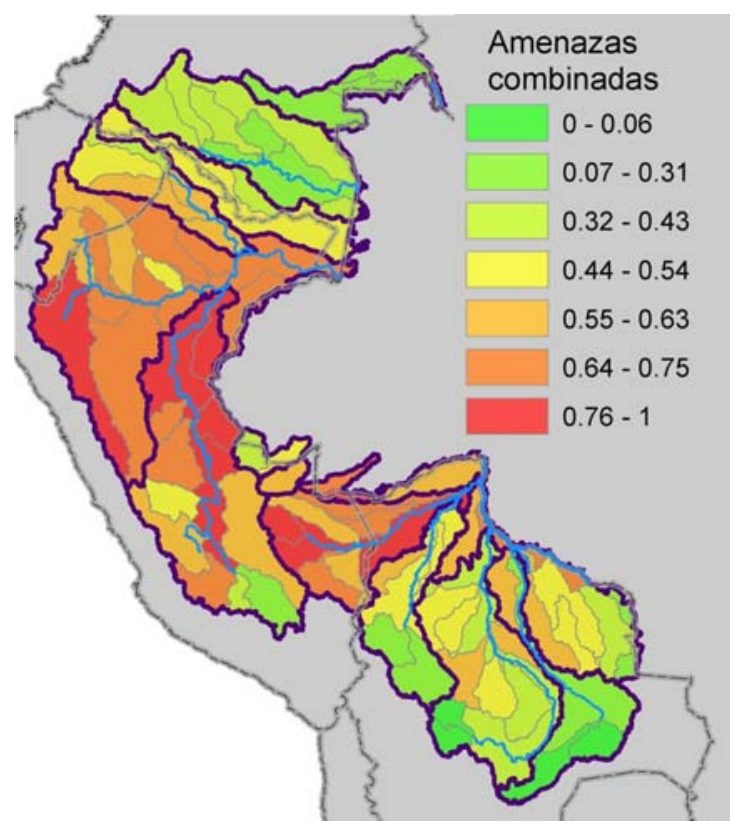

Figura 18. Incremento de las presiones sobre la biodiversidad, estimado hacia el 2030. Valores normalizados entre 0-1. Compuesto por el deterioro de la condición del paisaje, la conectividad hídrica y la deforestación potencial. 
remotos, como por ejemplo los datos sobre el almacenamiento de carbón, ya están disponibles en escalas de $1 \mathrm{~km}^{2}$. Se necesitan también a esta escala los datos vectoriales, como las áreas claves para la biodiversidad (Langhammer et al., 2007), compuesto por las áreas importantes para las aves (Devenish et al., 2009) y los sitios de la Alianza por la Extinción Cero (Ricketts et al., 2005). Con esto se podría esperar un mejoramiento significativo en la planificación al nivel local.

\section{Agradecimientos.}

Agradecemos a la Fundación John D. and Catherine T. MacArthur por proveer los recursos económicos necesarios para realizar este estudio. Reconocemos los esfuerzos de la UICN, BirdLife International, el Global Mammal Assessment y el Global Amphibian Assessment por proveer datos sobre el estado de conservación y distribución de los mamíferos, aves y anfibios. Además reconocemos a Lauren Raz por su apoyo con información sobre plantas en Colombia. Lily Paniagua y Nicole Sears ayudaron con la tarea de georreferenciar los especímenes de herbario. A Matt Finer y Clinton Jenkins por compartir sus datos sobre las represas hidroeléctricas de la región, lo mismo que datos georreferenciados sobre las concesiones mineras. A Juan Carlos Riveros y César F. Suárez por los datos sobre factores de presión. A Stefano Crema y sus estudiantes de Clark Labs, Nate Mietkiewicz y Julia Lenhardt por desarrollar el modelo sobre tasas futuras de deforestación.

\section{Literatura citada.}

Abell R., Thieme M., Revenga C., Bryer M., Kottelat M., Bogutskaya N., Coad B., Mandrak N., Contreras S., Bussing W., Stiassny M., Skelton P., Allen G., Unmack P., Naseka A., Ng R., Sindorf N., Robertson J., Armijo E., Higgins J., Heibel T., Wikramanayake E., Olson D., López H., Reis R., Lundberg J., Sabaj M. \& Petry P. 2008. Freshwater Ecoregions of the World: A new map of biogeographic units for freshwater biodiversity conservation. BioScience. 58(5): 403-414.

Alcamo J., Doll P., Henrichs T., Kaspar F., Lehner B., Rosch T. \& Siebert S. 2003. Development and testing of the Water GAP 2 global model of water use and availability. Hydrological Sciences Journal. 48(3): 31737.

Baccini A., Goetz S.J., Walker W.S., Laporte N.T., Sun M., Sulla-Menashe D., Hackler J., Beck P.S.A., Dubayah R., Friedl M.A., Samant S. \& Houghton R.A. 2012. Estimated carbon dioxide emissions from tropical deforestation improved by carbon-density maps. Nature Climate Change. 2: 182-185.

Barlow J. \& Peres C.A. 2008. Fire-mediated dieback and compositional cascade in an Amazonian forest. Philosophical Transactions of the Royal Society of London B Biol Sci. 363: 1787-1794.

Betts R.A., Malhi Y. \& Timmons Roberts J. 2008. The future of the Amazon: new perspectives from climate, ecosystem and social sciences. Philosophical
Transactions of the Royal Society of London B Biol Sci. 363: 1729-1735.

BirdLife International \& NatureServe. 2012. Bird species distribution maps of the world. BirdLife International, Cambridge, Reino Unido.

Brooks T.M., Mittermeier R.A., da Fonseca G.A.B., Gerlach J., Hoffmann M., Lamoreux J.F., Mittermeier C.G., Pilgrim J.D. \& Rodrigues A.S.L. 2006. Global biodiversity priorities. Science. 313: 58-61.

Bjorholm S., Svenning J.C., Skov F. \& Balslev H. 2005. Environmental and spatial controls of palm (Arecaceae) species richness across the Americas. Global Ecology and Biogegraphy. 14: 423-429.

Calderón E., Galeano G. \& García N. 2005. Libro rojo de plantas de Colombia. Volumen 3: Las bromelias, las labiadas y las pasifloras. Instituto de Investigación de Recursos Biológicos Alexander von Humboldt, Instituto de Ciencias Naturales, Universidad Nacional de Colombia, Bogotá, Colombia.

Comer P.J. \& Hak J. 2012. Landscape Condition in the Conterminous United States. Spatial Model Summary. NatureServe, Boulder, CO.

Convenio sobre la Diversidad Biológica. COP 10, Decisión X/2. http://www.cbd.int/decision/cop/?id=12268

CBD. 2010. Report of the Fourteenth Meeting of the Subsidiary Body on Scientific, Technical and Technological Advice. UN Environment Programme, Montreal, Canada.

Devenish C., Díaz Fernández D. F., Clay R. P., Davidson I. \& Yépez Zabala I. 2009. Important Bird Areas Americas - Priority sites for biodiversity conservation. BirdLife Conservation Series No. 16, BirdLife International, Quito, Ecuador.

Duellman W.E. 1999. Distribution patterns of amphibians in South America. En: Duellman W. E. (ed) Patterns of Distribution of Amphibians: 255-328. Johns Hopkins University Press, Baltimore, Maryland, USA.

Eastman J., Van Fossen M. \& Solarzano L. 2005. Transition potential modeling for land cover change. GIS, Spatial Analysis and Modeling. 357-386.

Ebeling J. \& Yasué M. 2008. Generating carbon finance through avoided deforestation and its potential to create climatic, conservation and human development benefits. Phil. Trans. R. Soc. B. 363: 1917-1924.

Edwards T.C., Scott J.M., Homer C.G. \& Ramsey R.D. 1993. Gap Analysis: A geographic approach for assessing national biological diversity. Natural Resources and Environmental Issues. 2: 65-71.

Finer M. \& Jenkins C.N. 2012. Proliferation of hydroelectric dams in the Andean Amazon and implications for Andes-Amazon connectivity. PLoS ONE 7(4): e35126. doi:10.1371/journal.pone.0035126

Finer M. \& Orta-Martínez M. 2010. A second hydrocarbon boom threatens the Peruvian Amazon: trends, projections, and policy implications. Environmental Research Letters (5). doi:10.1088/17489326/5/1/014012

Fitzhugh T.W. 2005. GIS Tools for Freshwater Biodiversity Conservation Planning. Transactions in GIS. 9(2): 247-263.

Friedlingstein P., Houghton R.A., Marland G., Hackler J., Boden T.A., Conway T.J., Canadell J.G., Raupach M.R., Ciais P. \& Le Quéré C. 2010. Update on CO2 emissions. Nature Geoscience. 3: 811-812. 
Fjeldså J. \& Rahbek C. 1998. Continent-wide conservation priorities and diversification processes. En: Mace G. M., Balmford A. \& Ginsberg J. R. (eds) Conservation in a changing world: 139-160. Cambridge Univ. Press, Cambridge, UK.

Fjeldså J., Alvarez M.D., Lazcano M. \& León B. 2005. Illicit crops and armed conflict as constraints on biodiversity conservation in the Andes region. Ambio. 34: 205-211.

Gärdenfors U., Hilton-Taylor C., Mace G.M. \& Rodríguez J.P. 2001. The application of IUCN Red List criteria at regional levels. Conservation Biology. 15: 1206-1212.

Gaston K.J. 2003. The Structure and Dynamics of Geographic Ranges. Oxford University Press, Oxford, Reino Unido.

Groves C. 2003. Drafting a conservation blueprint: a practitioner's guide to planning for biodiversity. Island Press.

Groves C., Valutis L., Vosick D., Neely B., Wheaton K., Touval J. \& Runnels B. 2000. Designing a Geography of Hope: A practitioner's handbook for ecoregional conservation planning. The Nature Conservancy, Arlington, VA.

Herzog S.K., Maillard O., Embert Z.D., Caballero P. \& Quiroga D. 2012. Range size estimates of Bolivian endemic bird species revisited: the importance of environmental data and national expert knowledge. Journal of Ornithology. 153: 1189-1202.

Houghton R.A. 2005. Tropical deforestation as a source of greenhouse gas emissions. En: Moutinho P. \& Schwartzman S. (eds) Tropical deforestation and climate change: 13-22. IPAM, Environmental Defense; Belém, Washington DC.

Jarvis A., Touval J.L., Castro M., Sotomayor L. \& Hyman G.G. 2009. Assessment of threats to ecosystems in South America. J Nat Conserv. 18: 180-188.

Josse C., Cuesta F., Navarro G., Barrena V., Cabrera E., Chacón-Moreno E., Ferreira W., Peralvo M., Saito J. \& Tovar A. 2009. Ecosistemas de los Andes del Norte y Centro. Bolivia, Colombia, Ecuador, Perú y Venezuela. Secretaría General de la Comunidad Andina, Programa Regional ECOBONA-Intercooperation, CONDESANProyecto Páramo Andino, Programa BioAndes, EcoCiencia, NatureServe, IAvH, LTA-UNALM, ICAEULA, CDC-UNALM, RUMBOL SRL. Lima, Perú.

Josse C. 2009. Mapa de ecosistemas de la Amazonía de Colombia, Ecuador, Perú y Bolivia. Reporte Técnico y Mapa elaborados con los aportes de Instituto Sinchi, EcoCiencia, IIAP y Rumbol. Programa BIOCAN, SGCAN.

Killeen T. J. 2007. A Perfect Storm in the Amazon Wilderness: Development and conservation in the context of the Initiative for the Integration of the Regional Infrastructure of South America (IIRSA). Center for Applied Biodiversity Science (CABS) Conservation International, Arlington, VA.

Knapp S. 2002. Assessing patterns of plant endemism in Neotropical uplands.The Botanical Review. 68: 22-37.

Köhler J., Vieites D.R., Bonett R.M., Hita García F., Glaw F., Steinke D. \& Vences M. 2005. New amphibians and global conservation: a boost in species discoveries in a highly endangered vertebrate group. Bioscience. 55: 693-696.
Langhammer P.F., Bakarr M.I., Bennun L.A., Brooks T.M., Clay R.P., Darwall W., De Silva N., Edgar G.J., Eken G., Fishpool L.D.C., Fonseca G.A.B. Da, Foster M.N., Knox D.H., Matiku P., Radford E.A., Rodrigues A.S.L., Salaman P., Sechrest W., \& Tordoff A.W. 2007. Identification and gap analysis of key biodiversity areas: targets for comprehensive protected area systems. IUCN, Gland, Suiza.

Larsen F.W., Londoño-Murcia M.C. \& Turner W.R. 2011. Global priorities for conservation of threatened species, carbon storage, and freshwater services: scope for synergy? Conservation Letters. 4: 355-363.

Lawler J.J., White D., Sifneos J.C. \& Master L.L. 2003. Rare species and the use of indicator groups for conservation planning. Conservation Biology. 17: 87582.

Le Quéré C., Raupach M.R., Canadell J.G., Marland G. et $a l^{*}$. 2009. Trends in the sources and sinks of carbon dioxide. Nature Geoscience. 2: 831-836. *Incluye 31 co-autores.

Lehner B., Verdin K. \& Jarvis A. 2008. New global hydrography derived from space-borne elevation data. Eos, Transactions, AGU. 89(10): 93-94.

León B., Roque J., Ulloa Ulloa C., Jørgensen P.M., Pitman N. \& Cano A. (eds) 2007. Libro Rojo de las plantas endémicas del Perú. Revista Peruana de Biología, Edición Especial. 13(2): 1-971.

León-Yánez S., Valencia R., Pitman N., Endara L., Ulloa Ulloa C. \& Navarrete H. (eds) 2011. Libro Rojo de las plantas endémicas del Ecuador. Publicaciones del Herbario QCA, Segunda Edición. Pontificia Universidad Católica del Ecuador, Quito.

Li W., Fu R., Negrón Juárez R.I \& Fernandes K. 2008. Observed change of the standardized precipitation index, its potential cause and implications to future climate change in the Amazon region. Philosophical Transactions of the Royal Society of London B Biol Sci. 363: 1767-1772.

Margules C.R. \& Pressey R.L. 2000. Systematic conservation planning. Nature. 405: 243-253

Millennium Ecosystem Assessment. 2005. Ecosystems and Human Well-being: Synthesis. Island Press, Washington, DC.

Ministerio de Medio Ambiente y Agua. 2012. Libro Rojo de la Flora amenazada de Bolivia. Vol. I. Zona Andina. La Paz. CMMAYA.

Myers N., Mittermeier R.A., Mittermeier G.C., da Fonseca G.A.B. \& Kent J. 2000. Biodiversity hotspots for conservation priorities. Nature. 403: 853-858.

Murdoch W., Ranganathan J., Polasky S. \& Regetz J. 2010.Using return on investment to maximize conservation effectiveness in Argentine grasslands. PNAS. 107(49): 20855-20862.

Naidoo R. \& X Iwamura V. 2007. Global-scale mapping of economic benefits from agricultural lands: implications for conservation priorities. Biological Conservation. 140: 40-49.

Nepstad D.C, Stickler C.M., Soares-Filho B. \& Merry. F. 2008. Interactions among Amazon land use, forest and climate: prospects for a near-term forest tipping point. Philosophical Transactions of the Royal Society of London B Biol Sci. 363: 1737-1746. 
Olson D.M. \& Dinerstein E. 2002. The Global 200: Priority ecoregions for global conservation. Ann. Missouri Bot. Gard. 89: 199-224.

Owens I.P.F. \& Bennett P.M. 2000. Ecological basis of extinction risk in birds: Habitat loss versus human persecution and introduced predators. PNAS. 97: $12144-12148$.

Petry P. \& Sotomayor L. 2009. Mapping Freshwater Ecological Systems with Nested Watersheds in South America. The Nature Conservancy.

Pitman N.C. \& Jørgensen P.M. 2002. Estimating the size of the world's threatened flora. Science. 298:989.

Programa de las Naciones Unidas para el Medio Ambiente (PNUMA) \& Organización del Tratado de Cooperación Amazónica (OTCA). 2009. Geo Amazonia. Perspectivas del medio ambiente en la Amazonía. PNUMA y OTCA.

RAISG. 2012. Amazonía bajo presión. Instituto Socioambiental, Sao Paulo.

Reeder D.M., Helgen K.M. \& Wilson D.E. 2007. Global trends and biases in new mammal species discoveries. Occasional Papers, Museum of Texas Tech University. 269: 135.

Reymondin L., Jarvis A., Perez-Uribe A., Touval J., Argote K., Rebetez J., Guevara E. \& Mulligan M. 2012. A methodology for near real-time monitoring of habitat change at continental scales using MODIS-NDVI and TRMM. Submitted Remote Sensing of Environment.

Ricketts T.H., Dinerstein E., Boucher T., Brooks T.M., Butchart S.H.M., Hoffmann M., Lamoreux J.F., Morrison J., Parr M., Pilgrim J.D., Rodrigues A.S.L., Sechrest W., Wallace G.E., Berlin K., Bielby J., Burgess N.D., Church D.R., Cox N., Knox D., Loucks C., Luck G.W., Master L.L., Moore R. Naidoo R., Ridgely R., Schatz G.E., Shire G., Strand H., Wettengel W. \& Wikramanayake E. 2005. Pinpointing and preventing imminent extinctions. PNAS. 102: 18497-18501.

Rodrigues A.S.L., Akçakaya H.R., Andelman S.J., Bakarr M.I., Boitani L., Brooks T.M., Chanson J.S., Fishpool L.D.C., Fonseca G.A.B. Da, Gaston K.J., Hoffmann M., Marquet P.A., Pilgrim J.D., Pressey R.L., Schipper J. Sechrest W. Stuart S.N. Underhill L.G., Waller R.W., Watts M.E.J. \& Yan X. 2004. Global gap analysis: Priority regions for expanding the global protected-area network. BioScience. 54: 1092-1100.

Rodrigues A.S.L. \& Brooks T.M. 2007. Shortcuts for biodiversity conservation planning: The effectiveness of surrogates. AREES 38: 713-737.

Rodríguez-Mahecha, J.V., Salaman P., Jørgensen P., Consiglio T., Suárez L., Arjona F. \& Bensted-Smith R. 2004. Tropical Andes. En: R.A. Mittermeier, P. RoblesGil, M. Hoffmann, J.D. Pilgrim, T.M. Brooks, C.G. Mittermeier \& G. Fonseca (eds) Hotspots Revisited: Earth's Biologically Richest and Most Endangered Ecoregions: 73-79. Second Edition. Cemex, Mexico.

Saatchi S.S., Harris N.L., Brown S., Lefsky M., Mitchard E., Salas W., Zutta B., Buermann W., Lewis S., Hagen S., Petrova S., White L., Silman M. \& Morel A. 2011. Benchmark map of forest carbon stocks in tropical regions across three continents. PNAS. 108: 9899-9904.

Schipper J., Chanson J.S., Chiozza F., Cox N.A. et al*. 2008. The status of the world's land and marine mammals: Diversity, threat, and knowledge. Science 322: 225-230. *Incluye 127 co-autores.
Sparks T., Butchart S., Balmford A., Bennun L., StanwellSmith D., Walpole M., Bates N., Bomhard B., Buchanan G., Chenery A., Collen B., Csirke J., Diaz R., Dulvy N., Fitzgerald C., Kapos V., Mayaux P., Tierney M., Waycott M., Wood L. \& Green R. 2011. Linked indicator sets for addressing biodiversity loss. Oryx 45(3): 411-419.

Stattersfeld A.J., Crosby M.J., Long A.J. \& Wege D.C. 1998. Endemic bird areas of the world. BirdLife International, Cambridge, UK.

Stuart S.N., Chanson J.S., Cox N.A., Young B.E., Rodrigues A.S. L., Fischman D.L. \& Waller R.W. 2004. Status and trends of amphibian declines and extinctions worldwide. Science. 306: 1783-1786.

Swenson J.J., Carter C.E., Domec J-C. \& Delgado C.I. 2011. Gold Mining in the Peruvian Amazon: Global prices, deforestation, and mercury imports. PLoS ONE 6(4): e18875. doi:10.1371/journal.pone.0018875.

Swenson J.J., Young B.E., Beck S., Comer P., Córdova J.H, Dyson J., Embert D., Encarnación F., Ferreira W., Franke I., Grossman D., Hernandez P., Herzog S.K., Josse C., Navarro G., Pacheco V., Stein B.A., Timaná M., Tovar A., Tovar C., Vargas J .\& Zambrana-Torrelio C.M. 2012. Plant and animal endemism in the Eastern Andean slope: Challenges to conservation. BMC Ecology.12: 1.doi: 10.1186/1472-6785-12-1.

Téllez, P., Petry P., Walschburger T., Higgins J. \& Apse C. 2011. Portafolio de Conservación de Agua Dulce para la Cuenca del Magdalena - Cauca. Programa NASCA, The Nature Conservancy \& Cormagdalena, Cartagena de Indias, Colombia.

The Nature Conservancy. 2007. Conservation Action Planning Handbook: Developing Strategies, Taking Action and Measuring Success at Any Scale. The Nature Conservancy, Arlington, VA.

Turner W.R., Brandon K., Brooks T. M., Gascon C., Gibbs H.K., Lawrence K. S., Mittermeier R.A. \& Sellig E.R. 2012. Global biodiversity conservation and the alleviation of poverty. BioScience. 62: 85-92.

United Nations Environmental Program (UNEP)- World Conservation Monitoring Centre (WCMC). 2009. International Expert Workshop on the 2010 Biodiversity Indicators and Post-2010 Indicator Development. UNEP-WCMC, Cambridge, UK; see http://www.cbd.int/doc/meetings/ind/emind02/official/emind-02-0709-10-workshop-report-en.pdf

Unión Internacional para la Conservación de la Naturaleza (UICN). 2001. IUCN Red List Categories: Version 3.1. Prepared by the IUCN Species Survival Commission. IUCN, Gland, Suiza y Cambridge, UK.

IUCN Red List of Threatened Species. Version 2012.1. UICN, Cambridge, Reino Unido. Disponible: http://www.iucnredlist.org.

Valencia R., León-Yánez S. \& Jørgensen P.M. 2000. Libro rojo de las plantas endémicas del Ecuador. Herbario QCA, Pontificia Universidad Católica del Ecuador, Quito, Ecuador.

Vié J.C., Hilton-Taylor C. \& Stuart S.N. 2008. Wildlife in a Changing World - An Analysis of the 2008 IUCN Red List of Threatened Species. UICN, Gland, Suiza.

Van der Werff H. \& Consiglio T. 2004. Distribution and conservation significance of endemic species of flowering plants in Peru. Biodiversity and Conservation. 13: 1699-1713. 
U.S. Geological Survey. 2000. HYDRO1k Elevation Derivative Database Cent. for Earth Resour. Obs. and Sci., Sioux Falls, SD, USA.
Young B.E., Franke I., Hernandez P.A., Herzog S.K., Paniagua L., Tovar C. \& Valqui T. 2009. Using spatial models to predict areas of endemism and gaps in the protection of Andean slope birds. Auk. 126: 554-565.

${ }^{1}$ NatureServe, 4600 N. Fairfax Dr. ,7 th Floor Arlington, VA 22203 USA

${ }^{2}$ NatureServe, Apdo. 358-1260, Plaza Colonial, San José, Costa Rica.

${ }^{3}$ NatureServe, 4600 N. Fairfax Dr. ,7 th Floor Arlington, VA 22203 USA.

${ }^{4}$ Global Policy and Programme Group-IUCN, Rue Muverney 28, 1196 Gland Switzerland.

${ }^{5}$ NatureServe, 4600 N. Fairfax Dr. ,7 th Floor Arlington, VA 22203 USA.

${ }^{6}$ NatureServe 2108 55th Street, Suite 220 Boulder, CO 80301 USA.

${ }^{7}$ The Nature Conservancy Latin America Conservation Region 73 Hideaway Lane Hollis, NH 03049 USA/Museum of Comparatuve Zoology, Harvard University, Cambridge M.A.

${ }^{8}$ Department of Bioscience Ecoinformatics \& Biodiversity Research Group, Aarhus University, Built $1540 \mathrm{Ny}$ Munkegade 114, 800 Asrhus C, Denmark.

${ }^{9}$ Missouri Botanical Garden, P.O. Box 299, St. Louis, MO 63166-0229 USA.

${ }^{10}$ Department of Aninal and Plant Sciences, The University of Sheffield, Western Bank.

${ }^{11}$ NatureServe 2108 55th Street, Suite 220 Boulder , CO 80301 USA.

${ }^{12}$ Missouri Botanical Garden, P.O. Box 299, St. Louis, MO 63166-0229 USA.

${ }^{13}$ Departamento de Ciencias, Fundación Amigos de la Naturaleza (FAN) Casilla 2241, Santa Cruz de la Sierra, Bolivia.

${ }^{14}$ Universidad Católica Boliviana "San Pablo", Carrera de Ingeniería Ambiental, Márquez esq. Parque J. Trigo Andia, Tupuraya, Cochabamba, Bolivia.

${ }^{16}$ Jet Propulsion Laboratory, Califorina Institute of Technology, 4800 Oak Grive Drive, Pasadena, CA. 91109 USA.

${ }^{17}$ Department of Bioscience Ecoinformatics \& Biodiversity Research Group, Aarhus University, Built $1540 \mathrm{Ny}$ Munkegade 114, 800 Asrhus C, Denmark.

${ }^{18}$ Facultad de Ciencias Forestales, Universidad Nacional Agraria La Molina, Apto 12-056, Lima 12, Perú.

${ }^{19}$ Observatorio Ambiental UAGRM Casilla 488 Santa Cruz de la Sierra, Bolivia. 